\title{
A Case Study on the Correction of Atmospheric Phases for SAR Tomography in Mountainous Regions
}

\author{
Muhammad Adnan Siddique, Student Member, IEEE, Tazio Strozzi, Senior Member, IEEE, Irena Hajnsek, Fellow, \\ IEEE, Othmar Frey, Senior Member, IEEE
}

\begin{abstract}
Synthetic aperture radar (SAR) tomography with repeat-pass acquisitions generally requires a priori phase calibration of the interferometric data stack by compensating for the atmosphere-induced phase delay variations. These variations act as a disturbance in tomographic focusing. In mountainous regions, the mitigation of these disturbances is particularly challenging due to strong spatial variations of the local atmospheric conditions and propagation paths through the troposphere. In this paper, we assess a data-driven approach to estimate these phase variations under a regression-kriging framework. The vertical stratification of the troposphere is modeled functionally while the impact of spatial turbulence is considered in a stochastic sense. The methodology entails an initial persistent scatterer interferometry (PSI) analysis. The atmospheric phases isolated for the persistent scatterers (PS) within the PSI processing are considered as samples of the 3-D distribution of the phase delay variations over the scene. These atmospheric phases are regressed against the spatial coordinates in map geometry at PS locations. In turn, kriging predictions are obtained at each location along the elevation profile where tomographic focusing is intended. A key point of this approach is that the requisite atmospheric corrections are incorporated within the tomographic focusing model. A case study has been performed on a data stack comprising 32 Cosmo-SkyMed stripmap images acquired over the Matter Valley in the Swiss Alps, in the summers of 20082013. The results show locally improved deformation sampling with tomographic methods compared to the initial PSI solution, primarily due to the improved phase calibration. In general, the work underscores the indispensability of height-dependent correction of atmospheric phases for SAR tomography.
\end{abstract}

Index Terms-Synthetic aperture radar (SAR), SAR tomography, atmospheric phases, persistent scatterer interferometry (PSI), tomography in mountainous regions, SAR interferometry, deformation analysis in mountainous areas, radar interferometry

\section{INTRODUCTION}

$\mathrm{S}_{\text {YNTHETIC aperture radar imaging involves two-way wave }}$ $S$ propagation through the atmosphere which acts as a refractive medium. The propagation is through both the troposphere and parts of the ionosphere for space-borne sensors in low earth orbit. Ionospheric effects on microwaves are frequency-dependent, and can often be ignored for highfrequencies such as the X-band [1], [2]. The optical path traversed by the waves in the troposphere is longer than the geometric path; therefore, additional phase delays are

Disclaimer: Graphical/textual differences may exist between this post-print and the final version published by IEEE which can be accessed at: https: //ieeexplore.ieee.org/document/8450633/. accumulated. The refractivity of the troposphere is mainly governed by temperature, pressure and water vapor [3], [4]. These factors typically vary in time and space - therefore, the refractivity changes over the scene as well as from one pass to the next, incurring variable phase delays which in general do not cancel out in interferogram formation leaving behind a phase footprint that we refer to as the atmospheric phase in this paper. These phases are typically a nuisance. If left uncorrected, the atmospheric phases appear as erroneous deformation in differential interferometric processing [5]-[8]. In case of SAR tomography [9]-[12], these phases act as a disturbance in focusing the scatterers in 3-D [13]-[17]. Therefore, prior to tomographic inversion, the interferometric data stack requires a precise phase calibration i.e. an estimation and compensation of the atmospheric phases in each interferogram.

The impact of the atmosphere is commonly distinguished in two aspects: firstly, the 3-D heterogeneities in refractivity caused by turbulent mixing; and secondly, a general decrease in refractivity with increasing altitude under normal atmospheric conditions, considering the lower troposphere to be a vertically stratified medium comprising thin layers that are horizontally homogeneous. In mountainous regions, both the turbulent mixing and vertical stratification contribute to variable phase delays in the interferograms, contrary to regions of flat topography where only turbulent mixing effects are relevant [3], [18]. The dominant part of the atmospheric phase is due to water vapor distribution [19]-[21], which is highly variable around the globe, and the more so in mountainous regions where the wind rises over or around the orography causing local overturning and condensation [22]. Therefore, the atmospheric phases in mountainous regions may exhibit strong spatial variations. In fact, correction of these phases remains a challenging problem.

\section{A. Motivation for SAR tomography in mountainous regions}

In case of urban areas, single-look SAR tomography has been proposed as an add-on to persistent scatterer interferometry (PSI) [23]-[27], with the objective of improving coverage of the PSI solution by separating the coherent scatterers in layover and simultaneously estimating their deformation parameters [28]-[31]. As such, layovers are generally more widespread in case of mountainous regions due to drastic variations in topography. And at the same time, often the slopes of interest in terms of critical mass movements happen 
to be in layover. Consequently, the coverage of PSI solution along such slopes remains limited [32], [33]. The use of SAR tomography as an add-on to PSI in mountainous regions though already at a loss given the typically low prevalence of coherent backscattering in these regions - can yet be rewarding as it may extend the coverage to slopes in layover. With this motivation, we intend to investigate the possibility of using SAR tomography in an alpine region. However, an immediate concern is how to achieve the requisite phase calibration.

\section{B. Related work from SAR interferometry}

Several investigations have addressed atmospheric phases since the first reports [5], [19], [20], [34], albeit only in the context of SAR interferometry. The methods proposed to correct these phases can be broadly categorized into those which utilize auxiliary information from external sources to estimate the phase delays, and those which are purely datadriven. In the former category, the commonly used auxiliary information are the output of numerical weather models, such as the Weather Research and Forecasting (WRF) model [35] and the global atmospheric reanalysis data from the ERAInterim project [36]. The hindcast of the relevant meteorological parameters from the weather models is used to estimate 3-D field of refractivity of the atmosphere at the time of SAR acquisitions. These estimates are subsequently used to generate synthetic phase delay maps to compensate for the atmospheric effects [37], [38]. The usefulness of the weather models is often questionable since the resolution of the weather data is too coarse and they are generally not concurrent with the SAR acquisitions [39]. Global Navigation Satellite Systems (GNSS) measurements can also be used as auxiliary information. In general, the wet zenith delays measured with GNSS measurements are converted to delays in slant range followed by spatial interpolation over the area imaged by SAR [40]. Several techniques for the assimilation of GNSS measurements into interferometric processing have been proposed over time [40]-[43]. GNSS-based approach is generally effective, but its application remains limited as permanent GNSS stations are often absent or sparsely distributed for most areas around the world.

In the absence of or unsuitability of external sources due to the aforementioned limitations, purely data-driven alternatives are commonplace. They work directly on the interferometric data, customarily adopting spatio-temporal filtering to isolate the phase variations typically signified by atmosphere relative to other contributors such as deformation, residual topography as well as those occurring due to the multiplicity of the scatterers (in the context of tomography). The phase variations variations are typically considered to be spatially correlated up to a certain extent and uncorrelated in time [23], [24], [44]-[46] (especially in case of repeat-pass spaceborne acquisitions). In general, a network of several interferograms is desired to assess statistical properties of the different sources of phase variations. Any filtering in time should preserve nonlinear deformation, if any, as emphasized by several studies [43]-[45], [47]. Moreover, temporally phase coherent targets, i.e. persistent scatterers (PS) [23], [24], [48] are needed to reliably extract the different phase contributions and to restrict the propagation of decorrelation noise during interferometric processing (phase filtering, unwrapping, etc.). The atmospheric phases estimated for the PS are low pass filtered and spatially interpolated over the scene, for example with a kriging interpolator [6], [23].

A linear dependence of interferometric phases on topography is adopted in several studies to model a vertically stratified atmosphere [49]-[52]. The investigation in [53] suggests a power-law model as a generalization of the linear model. While the topography-dependent part of atmospheric phases is typically modeled functionally, the contribution of turbulent mixing is often dealt with in a stochastic sense. The spatial heterogeneity in the atmospheric signal is modeled as a random process. The second-order statistics of the process are estimated by transforming the spatial spectra to corresponding spatial structure or covariance functions [3], [54], [55]. Under Kolmogorov turbulence theory in isotropic conditions, the spectrum and the associated covariance function theoretically follow a power-law distribution [19], [40]. In practice, spectra are quite variable and may not always be structured according to the Kolmogorov theory [3], [56]. The power-law exponent varies in different scaling regimes, and needs to be empirically estimated [41], [57]. As the lag between the observation points increases, the variance among them increases. In this way, an empirical variance-covariance matrix can be built up for data quality description as well as to constrain underlying geophysical processes that require the covariance of the noise [56], [58], [59]. Moreover, it can be used to improve spatial interpolation of the atmospheric phases from the observed locations (whether individual GNSS sites, weather stations or PS locations where we have already estimated the atmospheric phases) towards the unobserved locations in the scene [60][62]. In [62], a 2-D kriging interpolation in easting-northing with a linear semivariogram model is used. The results obtained are shown to be better than spline interpolation.

\section{Research gaps}

To date, all atmospheric phase correction approaches estimate a single correction for each pixel. When the topographydependence is modeled, this correction is estimated at the specific height for the given pixel. In the context of tomography in mountainous regions, multiple coherent scatterers may lie in the same pixel while being separated in height by as much as a kilometer or more, as shown in Fig. 1. Due to the difference in the vertical refractivity profiles of the troposphere at each epoch relative to the master acquisition, the atmospheric phase delays experienced by each scatterer can be very different. While a single correction may work for one of the scatterers, the uncorrected phase delays for the second or more scatterers would prevail as noise and may hamper tomographic focusing. Consequently, modeling vertical stratification of the troposphere within the same pixel is required. Moreover, due to the side-looking geometry of the SAR sensors, the large difference in height between the scatterers in layover results in comparably large lateral separation among the scatterers. Therefore, a method that corrects for atmospheric phases for 
scatterers at different height levels must also account for possible lateral variations. In turn, any spatial interpolation should weight over observation points from local physical neighborhoods of each scatterer.

\section{Contributions \& limitations of this paper}

Deformation assessment using differential tomography has thus far been applied in urban regions or specific infrastructure where the underlying topography is generally flat [14], [31], [63]-[65]. This investigation is a first step towards facilitating its application in rugged mountainous terrain by providing the following specific contributions filling in the aforementioned research gaps:

1) A data-driven, regression-kriging framework is presented to model both vertical stratification and mixing effects.

2) For a given layover-affected pixel, multiple corrections are applied for potentially multiple scatterers at different positions along the elevation axis.

3) These corrections are incorporated within tomographic focusing at each 3-D point of interest prior to inversion and scatterer detection.

At the same time, this paper does not specifically address some other potential limitations on the performance of tomography in mountainous regions. These include the possibility of range migration of the target from a given range bin to the adjacent bin when the tomographic focusing is performed over very large elevation extents [31], [64]. The severity of the problem depends on the baseline distribution as well as on the magnitude of topography changes. Another potential limitation is incurred as an aftermath of topographyinduced radiometric effects [66], [67]. The physical pixelarea in layover and foreshortening-affected pixels may be too large to allow point-like scattering, which in turn reduces the prevalence of coherent scatterers.

\section{E. Outline}

The methodology proposed in this work entails a prior PSI analysis. The Interferometric Point Target Analysis (IPTA) [24] toolbox is used to iteratively identify a set of PS (which are single dominant scatterers). The atmospheric phases for these PS are estimated within the PSI processing by isolating these components from other phase contributions (e.g. residual topography and deformation) with appropriate spatiotemporal filtering. The PS are geocoded using an external digital elevation model (DEM). We consider that these phases represent samples of the 3-D distribution of the atmospheric phase delay variations over the entire scene in map geometry. The atmospheric phases are regressed against easting, northing and height at the PS locations. The statistics of the residue of the regression fit are subjected to a variogram analysis. The empirical semivariogram for each interferometric layer in the stack is fit with a parametric model, and the corresponding covariance function is computed. For each range-azimuth pixel, and for each discrete location along the elevation axis where tomographic inversion is intended, the 3-D location in terms of

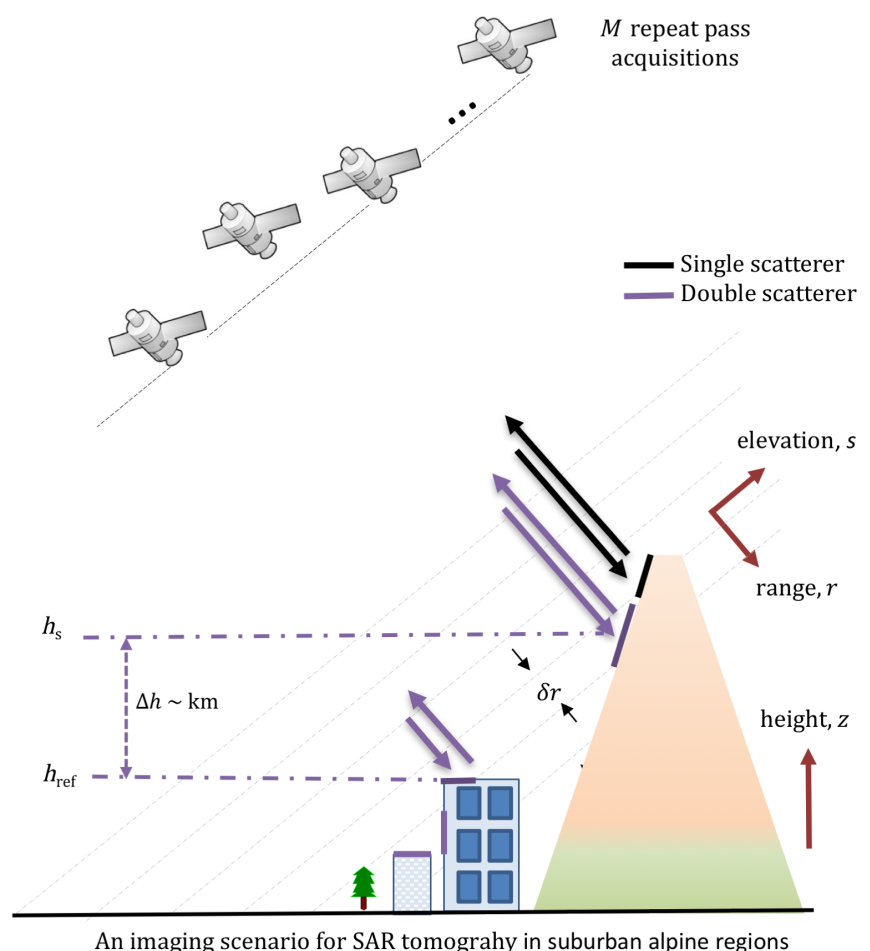

Figure 1. An imaging scenario for spaceborne SAR tomography in mountainous regions with multi-baseline repeat pass acquisitions. The total backscatter in a given range-azimuth resolution cell $(\delta r)$ may comprise of multiple contributions along the elevation axis.

range-azimuth-elevation is projected into map coordinates and a best linear unbiased predictor under universal kriging [68] is used to predict the atmospheric phase for that specific location. Single-look beamforming is used for differential tomographic inversion to retrieve the $2 \mathrm{D}$ reflectivity (as a function of the elevation and deformation velocity). A generalized likelihood ratio test is used to detect single and double scatterers in the scene.

The remainder of this paper is organized as follows. Section II discusses the implication of height-dependence of the atmospheric phases for SAR tomography. Section III describes the methodology proposed in this investigation. The characteristics of the interferometric data stack used in the investigation are given in Section IV. Results are provided in section $\mathrm{V}$, and a detailed discussion follows in section VI.

\section{MODELING VERTICALLY STRATIFIED ATMOSPHERE FOR SAR TOMOGRAPHY}

The dimensions of a SAR range-azimuth resolution cell are nowadays on the order of a few meters or tens of meters. They are small compared to the scales at which lateral variations in atmospheric phases occur, i.e. several hundreds of meters to kilometers [3]. Therefore, within a given resolution cell, lateral variations are not expected. However, vertical stratification effects within the same resolution cell may not be ignorable especially in mountainous regions when the multiple scatterers superposing in the cell may be separated by a kilometer or so, as shown in Fig. 1. In this section, first we consider 
the tomographic model in the absence of atmospheric phases, followed by a discussion on the impact of vertical stratification and its mathematical modeling.

\section{A. SAR tomography}

In the absence of any additive or multiplicative noise, classical SAR tomography (3-D SAR) relates the single-look complex SAR signal, $y_{m}$ for a given range-azimuth resolution cell, with the unknown reflectivity profile $\alpha(s)$ according to the following mathematical model [64], [69]-[71]:

$$
y_{m}=\int_{\mathcal{I}_{s}} \alpha(s) \exp \left[-j \varphi_{m}(s)\right] \mathrm{d} s
$$

where $s$ denotes the elevation axis, $\mathcal{I}_{s}$ is the observed elevation extent, and the interferometric phase, $\varphi_{m}(s)$ models the phase variations along the elevation due to sensor-to-target geometry as follows:

$$
\varphi_{m}(s)=2 k \triangle r_{m}(s)
$$

where $k=2 \pi / \lambda$ is the central wavenumber and $\triangle r_{n}(s)$ is the path-length difference:

$$
\triangle r_{m}(s)=r_{m}(s)-r_{0}(s) \approx \frac{s^{2}}{2\left(r_{0}-b_{m}^{\|}\right)}-\frac{b_{m}^{\perp} s}{r_{0}-b_{m}^{\|}} .
$$

The variable $r$ represents the range distance from the sensor to the target location, and the subscript $m$ refers to the interferometric pair in consideration. The interferograms are set up relative to a single master acquisition denoted with $m=0$. The orthogonal and parallel components of the $m^{\text {th }}$ spatial baseline are $b_{m}^{\perp}$ and $b_{m}^{\|}$, respectively.

The interferometric phase model for 3-D SAR assumes that no scatterer in the resolution cell has undergone any displacement during the time span between the first and the last acquisition in the data stack. If, however, they has been any displacement, there would be additional phase variations which must be accounted for in the phase model. Assuming that the motion is a temporally linear displacement in the line of sight (LOS), differential tomography [28]-[30] (4D SAR) extends the phase model as follows:

$$
\varphi_{m}(s, \nu)=2 k\left[\triangle r_{m}(s)+v t_{m}\right]
$$

where $v$ is the (average) linear deformation velocity of the scatterer, and $t_{m}$ is the temporal baseline for the $m^{\text {th }}$ interferogram. The SAR signal can now be rewritten as [71]-[73]:

$$
y_{m}=\iint_{\mathcal{I}_{s} \mathcal{I}_{v}} \alpha(s, v) \exp \left[-j \varphi_{m}(s, v)\right] \mathrm{d} s \mathrm{~d} v
$$

where $\mathcal{I}_{v}$ is the extent of the expected linear deformation velocity.

\section{B. Modeling atmospheric phases due to stratified troposphere}

The standard model for atmosphere considers the refractivity, $N$ of the troposphere to be exponentially decaying with increasing height [4], [43], [52]:

$$
N(z)=N^{\mathrm{sl}} \exp \left(-\frac{z}{H}\right)
$$

where $z$ represents the height above sea level (a.s.l.), $H$ is the decay parameter typically set at $7.35 \mathrm{~km}$, and $N^{\text {sl }}$ indicates the refractivity at sea-level. While $H$ is typically assumed to be constant, the temporal variations of the refractivity from one SAR acquisition to the next can be associated with time-dependence of $N^{\text {sl }}$ [43]. With these considerations, the interferometric phase delay due to refractivity change between the acquisitions forming the $m^{\text {th }}$ interferogram, for a scatterer at a reference height, $h_{\text {ref }}$ in the valley (see Fig. 1 ), is given as [3]:

$$
\psi_{m}^{h_{\text {ref }}}=10^{-6} \frac{2 k}{\cos \theta_{\text {inc }}} \int_{h_{\text {ref }}}^{h_{\text {sat }}} \Delta N_{m}^{\mathrm{sl}} \exp (-z / H) \mathrm{d} z .
$$

$\theta_{\text {inc }}$ is the angle of incidence and $h_{\text {sat }}$ is the height of the satellite bearing the sensor. They are considered here to be the same for each pass. If there is a second scatterer at an arbitrary height $h_{s}$, though in the same range-azimuth resolution cell, additional phase variations will be incurred relative to the reference height:

$$
\begin{aligned}
\Phi_{m}^{h_{s}, h_{\text {ref }}} & =10^{-6} \frac{2 k}{\cos \theta_{\text {inc }}}\left(\int_{h_{s}}^{h_{\text {sat }}} \Delta N_{m}^{\text {sl }} \exp (-z / H) \mathrm{d} z\right. \\
& \left.-\int_{h_{\text {ref }}}^{h_{\text {sat }}} \Delta N_{m}^{\mathrm{sl}} \exp (-z / H) \mathrm{d} z\right) .
\end{aligned}
$$

Using the first two terms of the Taylor series expansion of $\exp (-z / H)$ at $z=h_{s}$, and that $\exp (-z / H) \rightarrow 0$ as $z \rightarrow$ $h_{\text {sat }}$ (which is in agreement with the fact that the relative tropospheric delays converge to zero typically around 7-13 $\mathrm{km}$ [53]), the expression in eq. (8) is simplified as follows:

$$
\Phi_{m}^{h_{s}, h_{\mathrm{ref}}} \approx-10^{-6} \frac{2 k}{\cos \theta_{\text {inc }}} \Delta N_{m}^{\mathrm{sl}} \exp \left(-h_{\mathrm{ref}} / H\right) \cdot\left(h_{s}-h_{\mathrm{ref}}\right) .
$$

The atmospheric phase for the scatterer at $h_{s}$ is:

$$
\psi_{m}^{h_{s}}=\psi_{m}^{h_{\mathrm{ref}}}+\Phi_{m}^{h_{s}, h_{\mathrm{ref}}} \approx \dot{\beta}_{0}+\dot{\beta}_{1} \cdot \Delta h
$$

where the constants $\hat{\beta}_{0}$ and $\hat{\beta}_{1}$, following eq. (7) and eq. (9) respectively, are given by

$$
\begin{aligned}
& \dot{\beta}_{0}=10^{-6} \frac{2 k}{\cos \theta_{\text {inc }}} \Delta N_{m}^{\mathrm{sl}} H \exp \left(-h_{\mathrm{ref}} / H\right) \\
& \dot{\beta}_{1}=-10^{-6} \frac{2 k}{\cos \theta_{\text {inc }}} \Delta N_{m}^{\mathrm{sl}} \exp \left(-h_{\mathrm{ref}} / H\right) .
\end{aligned}
$$

$\triangle h=h_{s}-h_{\text {ref }}$ is the height difference between the scatterers, and its projection in the elevation axis is $\triangle s=\triangle h \sin ^{-1} \theta_{\text {inc }}$.

The expressions in eq. (10) and (11) indicate that apart from dependence on the wavelength and incidence angle, the atmospheric phases depend both on the magnitude of the unknown refractivity change $\left(\triangle N_{s}\right)$ as well as the difference in elevation $(\triangle s)$ between the scatterers in layover. Even if we have an accurate estimate of the atmospheric phase at the reference height (e.g. with phases reliably measured over a corner reflector, or with a GNSS station in immediate proximity, or with local spatial filtering over several PS located in the built-up valley) and compensate for it, the additional 
phase variations due to the second scatterer at the higher altitude will remain uncorrected.

To model atmospheric phases in differential tomography, the mathematical model in eq. (5) is rewritten as:

$$
y_{m}^{p d}=\iint_{\mathcal{I}_{s} \mathcal{I}_{v}} \alpha(s, v) \exp \left[-j \psi_{m}(s)\right] \exp \left[-j \varphi_{m}(s, v)\right] \mathrm{d} s \mathrm{~d} v
$$

where $y_{m}^{p d}$ is the SAR signal observed in the presence of atmospheric phases. It is assumed here that the refractivity changes are uncorrelated with the temporal baselines. In fact the refractivity changes are random from one interferogram to the next. Therefore, uncompensated atmospheric phases will act as a random phase disturbance and hamper tomographic focusing. It is not within the scope of this paper to describe the effect of these disturbances in a statistical sense; interested readers are referred to [13].

\section{Methodology}

The methodology adopted in this work is a data-driven approach to correct for the atmospheric phases and to apply differential SAR tomography on a data stack of repeat pass single-reference interferograms over a mountainous region. Fig. 2 shows the overall methodology in a flowchart, while the details of the different processing steps involved are given in the following subsections.

\section{A. PSI processing}

PSI processing is performed using the Interferometric Point Target Analysis (IPTA) [24], [45], [74] framework. The preprocessing includes selection of a reference acquisition and coregistration of the data stack. A multi-look intensity image of the reference acquisition is geocoded using an external digital elevation model (DEM). DEM heights are transformed into radar geometry, and used in the coregistration of all the acquisitions to the reference. The overall coregistration process includes a last refinement step using the fine offsets estimated between the acquisitions [45], [66], [67].

An initial list of PS candidates is prepared on the basis of high temporal stability of the backscattering and low spectral diversity. These two criteria are proxies to identify single dominant scatterers with point-like response. In alpine regions, such scatterers are mostly man-made structures in the valley or occasional bare rocky patches on the mountainsides and tops. A reference point is selected, and double-differenced point differential interferograms are computed. The observed interferometric phase in each layer of the stack is modeled as the sum of atmospheric phase, phase variations due to residual topography and deformation, and phase noise (decorrelation or miscalibration). To separate these phase contributions, an iterative least-squares bivariate regression is applied exploiting the spatial and temporal baselines to obtain estimates of residual topography and linear deformation velocity. The dispersion of the residual phase is used as a quality metric. The PS candidates for which the standard deviation of the residual phase is higher than a preselected threshold are rejected. We consider several acquisitions are available to justify the

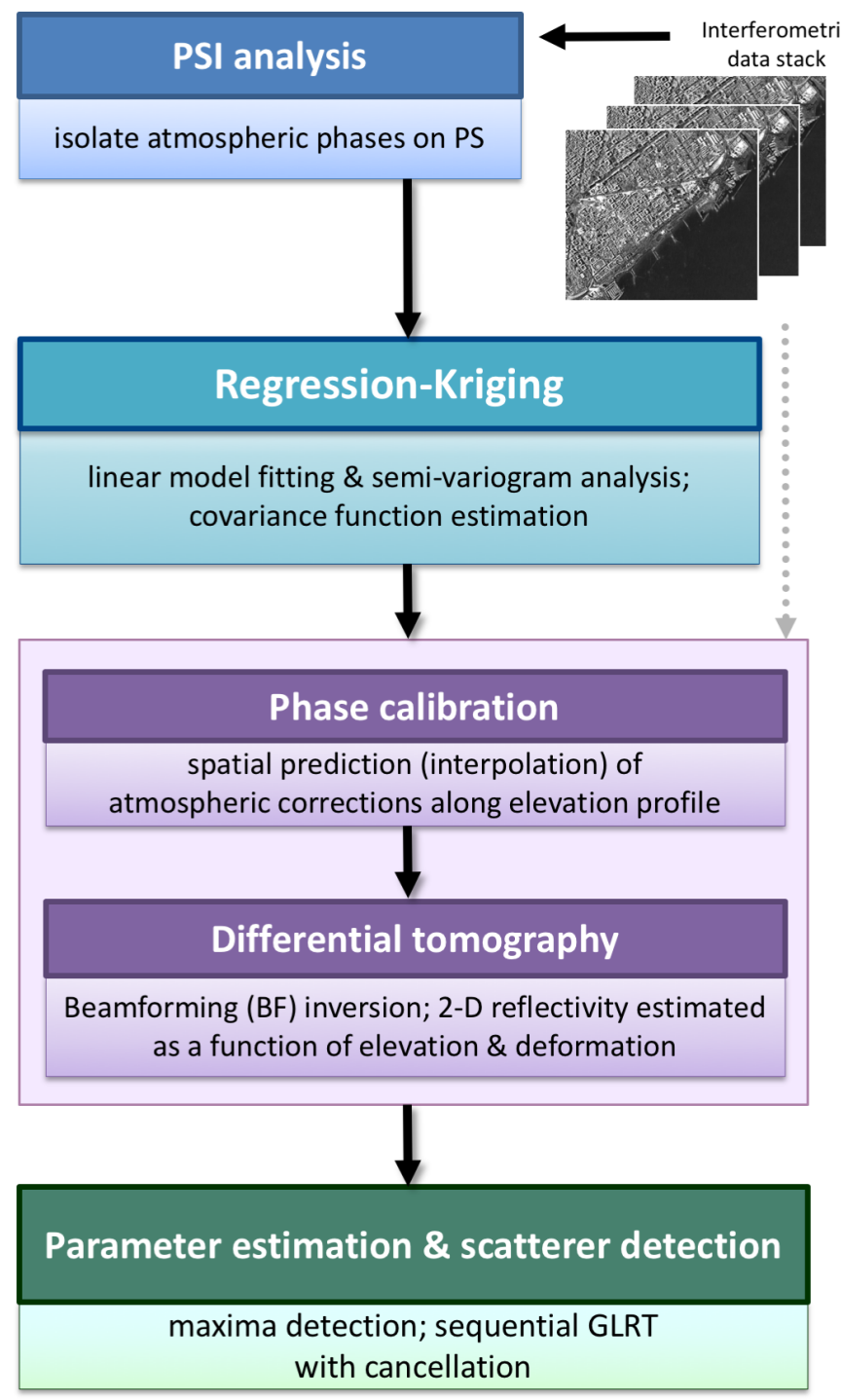

Figure 2. Flowchart of the processing methodology.

assumption that the atmospheric phases are uncorrelated with other phase contributions. The residue of the fit is spatially filtered over the retained PS candidates in small local neighborhoods and unwrapped. The unwrapped phase is fit with a linear model for height-dependence, as in eq. 10, using DEM heights. The model fit is subsequently tried over more candidates. The aforementioned processing steps are repeated in several iterations, until the solution has converged i.e. no substantial changes in the estimated parameters (atmospheric phases, residual topography, linear deformation) occur in further iterations, nor do more PS are identified with the same quality restrictions.

For more details on various interferometric processing strategies using the IPTA toolbox, the interested readers are referred to earlier works [24], [45], [74]-[76].

\section{B. Regression-kriging}

The PS identified in the interferometric processing are geocoded. We consider the atmospheric phases estimated for 
the PS as samples of the physical 3-D distribution of the atmospheric signal over the scene. Considering the possibility of lateral variations besides vertical stratification effects, we model the unwrapped atmospheric phases for a given interferometric layer from the stack with the following multiple linear regression model:

$$
\begin{aligned}
\psi(\boldsymbol{x}) & =\mathbf{x}^{T} \boldsymbol{\beta}+\varepsilon(\boldsymbol{x}) \\
& =\left[\begin{array}{llll}
1 & x_{e} & x_{n} & h
\end{array}\right]\left[\begin{array}{c}
\beta_{0} \\
\beta_{1} \\
\beta_{2} \\
\beta_{3}
\end{array}\right]+\varepsilon(\boldsymbol{x})
\end{aligned}
$$

where $\boldsymbol{x} \triangleq\left(x_{e}, x_{n}, h\right)$ represents a general 3-D location in map geometry in terms of easting, $x_{e}$, northing, $x_{n}$ and height, $h . \beta$ terms are the regression coefficients. $\boldsymbol{x}=\mathbb{T}\{r, a, s\}$ where $\mathbb{T}\{$.$\} is the geocoding transformation applied on a$ range-azimuth-elevation tuple, $(r, a, s)$. The linear dependence on map coordinates attempts to extract 'trends' in the atmospheric phases, lateral as well as vertical. Within the interferometric layer, they are treated in a deterministic sense. The non-linear heterogeneity owing to tropospheric mixing effects is reflected in the residue, $\varepsilon(\boldsymbol{x})$, which we treat as stochastic. We assume it to be a zero-mean, spatially correlated, secondorder stationary process. The variogram of such a process is defined as [77]:

$$
\gamma(\boldsymbol{\ell})=\frac{1}{2} E\left[\{\varepsilon(\boldsymbol{x})-\varepsilon(\boldsymbol{x}+\boldsymbol{\ell})\}^{2}\right]
$$

where $\ell$ is the spatial lag between two locations. Under this assumption, the variance of the process is considered to be a constant, while the spatial correlation does not depend on the location itself but only on the lag between the locations. Moreover, in case of a second-order stationary processes, the variogram and the covariance function are equivalent [77]:

$$
\gamma(\ell)=C(0)+C(\ell)
$$

where $C(0)=\sigma^{2}$ is the variance of the process. We further assume isotropy i.e. direction independence of the semivariance of $\varepsilon(\boldsymbol{x})$. It implies that the spatial correlation depends only on the magnitude of the lag, $\ell=\|\ell\|$, which is the Euclidean distance between two locations in map geometry in our case.

Sample variogram estimation: The atmospheric phases computed for the PS are regressed on their 3-D map coordinates for each interferometric layer, and the estimated residuals, $\hat{\varepsilon}(\boldsymbol{x})$ are used to obtain the sample variogram as follows [68]:

$$
\hat{\gamma}\left(\tilde{\ell}_{j}\right)=\frac{1}{2 \mathcal{N}(\ell)} \sum_{i=1}^{\mathcal{N}(\ell)}\left\{\hat{\varepsilon}\left(\boldsymbol{x}_{i}\right)-\hat{\varepsilon}\left(\boldsymbol{x}_{i}+\hat{\boldsymbol{\imath}} \ell\right)\right\}^{2}, \quad \forall l \in \tilde{\ell}_{j}
$$

where $\hat{\varepsilon}\left(\boldsymbol{x}_{i}\right)$ is the residue for the $i^{\text {th }}$ PS, $\hat{\varepsilon}\left(\boldsymbol{x}_{i}+\hat{\boldsymbol{\imath}} \ell\right)$ is the residue for a PS that is located such that the radial distance between the location pair $\left\{\left(\boldsymbol{x}_{i}\right),\left(\boldsymbol{x}_{i}+\hat{\boldsymbol{\imath}} \ell\right)\right\}$ is within the given distance interval, $\tilde{\ell}_{j}$ and $\mathcal{N}(\ell)$ is the number of such paired comparisons. $\hat{\imath}$ represents a unit vector in any direction. The subscript $j$ indicates the index over the distance intervals used to compute the sample variogram.
Spatial prediction: The regression-kriging a.k.a. universal kriging-based best linear unbiased predictor (BLUP) of the atmospheric phase at a $3-\mathrm{D}$ location $\boldsymbol{x}_{0}$ is [68]:

$$
\hat{\psi}\left(\boldsymbol{x}_{0}\right)=\mathbf{x}_{0}^{T} \hat{\boldsymbol{\beta}}+\mathbf{v}^{T} \boldsymbol{V}^{-1}(\boldsymbol{\Psi}-\mathbf{X} \hat{\boldsymbol{\beta}})
$$

where $\mathbf{X}$ is the design matrix and $\boldsymbol{\Psi}$ is the vector of the atmospheric phases at PS locations:

$$
\mathbf{X}=\left[\begin{array}{c}
\mathbf{x}_{1}^{T} \\
\mathbf{x}_{2}^{T} \\
\vdots \\
\mathbf{x}_{N_{\mathrm{ps}}}^{T}
\end{array}\right], \quad \boldsymbol{\Psi}=\left[\begin{array}{c}
\psi\left(\boldsymbol{x}_{1}\right) \\
\psi\left(\boldsymbol{x}_{2}\right) \\
\vdots \\
\psi\left(\boldsymbol{x}_{N_{\mathrm{ps}}}\right)
\end{array}\right]
$$

$N_{\mathrm{ps}}$ is the number of PS used for kriging. The regression coefficients can be estimated with generalized least squares [68]:

$$
\hat{\boldsymbol{\beta}}=\left(\mathbf{X}^{T} \mathbf{V}^{-1} \mathbf{X}\right)^{-1} \mathbf{X}^{T} \mathbf{V}^{-1} \mathbf{\Psi}
$$

A parametric variogram model is fit to the sample variogram to get a smooth function that is continuous for all non-zero lags. The model selected must also ensure that the spatial predictions are associated with non-negative variances [68], [77]. Examples of models commonly used in the field of Geostatistics are spherical, exponential, power law, Gaussian, Matérn, etc. [78]. Using eq. (15), the model fit is used to estimate the covariance function, which in turn allows the estimation of the data covariance matrix $\mathbf{V}$ (using the pairwise lags among the PS), and the covariance vector $\mathbf{v}$ (using the lags between the location $\boldsymbol{x}_{0}$ and the locations of the PS).

The prediction error variance for the predictor in eq. 17 is [68]:

$$
\sigma_{e}^{2}\left(\boldsymbol{x}_{0}\right)=\sigma^{2}-\mathbf{v}^{T} \mathbf{V}^{-1} \mathbf{v}+\zeta\left(\mathbf{X}^{T} \mathbf{V}^{-1} \mathbf{X}\right)^{-1} \zeta^{T}
$$

where $\zeta=\mathbf{x}_{0}^{T}-\mathbf{v}^{T} \mathbf{V}^{-1} \mathbf{X}$. The second term in eq. 20, $\mathbf{v}^{T} \mathbf{V}^{-1} \mathbf{v}$ is equal to $\sigma^{2}$ when $\boldsymbol{x}_{0}$ is identical to an observed location, i.e. a PS location in $\mathbf{X}$. The expression $\left(\mathbf{X}^{T} \mathbf{V}^{-1} \mathbf{X}\right)^{-1}$ in the third term becomes zero when $\boldsymbol{x}_{0}$ is an observed location, and it increases otherwise when $\boldsymbol{x}_{0}$ gets more distant from observed locations in $\mathbf{X}$. Therefore, the prediction of atmospheric phases becomes less precise at locations farther from the PS distribution available/used in the kriging setup.

\section{Tomographic focusing}

Considering we have $M$ acquisitions, the observed SAR signal vector for a given range-azimuth pixel $(r, a)$ is

$$
\mathbf{y}^{p d}=\left[\begin{array}{llll}
y_{0}^{p d} & y_{1}^{p d} & \ldots & y_{M-1}^{p d}
\end{array}\right]^{T} .
$$

The observed elevation and deformation extents, $\mathcal{I}_{s}$ and $\mathcal{I}_{v}$, respectively, are discretized. Inverting the differential tomographic model in eq. (12), the $2 \mathrm{D}$ scatterer reflectivity at the discrete pair $\left(s_{i}, v_{j}\right)$ is focused using single-look beamforming (BF) as follows:

$$
\hat{\alpha}\left(s_{i}, v_{j}\right)=\frac{1}{M} \mathbf{a}^{H}\left(s_{i}, v_{j}\right) \mathbf{y}^{p d} .
$$


The steering vector $\mathbf{a}\left(s_{i}, v_{j}\right)$ is set up such that atmospheric phase correction is incorporated within the tomographic focusing at each discrete point of interest along the elevation axis, as shown below:

$$
\begin{aligned}
& \mathbf{a}\left(s_{i}, v_{j}\right)= \\
& {\left[\begin{array}{c}
\exp \left[-j\left\{\begin{array}{l}
\left.\varphi_{0}\left(s_{i}, v_{j}\right)+\hat{\psi}_{0}\left(\mathbb{T}\left\{r, a, s_{i}\right\}\right)\right\} \\
\exp \left[-j\left\{\varphi_{1}\left(s_{i}, v_{j}\right)+\hat{\psi}_{1}\left(\mathbb{T}\left\{r, a, s_{i}\right\}\right)\right\}\right.
\end{array}\right]\right. \\
\vdots \\
\exp \left[-j\left\{\varphi_{M-1}\left(s_{i}, v_{j}\right)+\hat{\psi}_{M-1}\left(\mathbb{T}\left\{r, a, s_{i}\right\}\right)\right\}\right]
\end{array}\right] .}
\end{aligned}
$$

\section{Scatterer parameter estimation and detection}

For a given range-azimuth resolution cell, we make the following hypotheses. It contains no coherent scatterer $-\mathcal{H}^{0}$; it comprises a single dominant scatterer $-\mathcal{H}^{1}$, or it is a double scatterer $-\mathcal{H}^{2}$. The unknown scatterer parameters (elevation and deformation velocity) for a potential first and second scatterer are estimated with the following beamforming-based maximization [29], [31], respectively:

$$
\begin{gathered}
\left(\hat{s}_{1}, \hat{v}_{1}\right)=\underset{\left(s_{i}, v_{j}\right) \in\left(\mathcal{I}_{s}, \mathcal{I}_{v}\right)}{\arg \max }\left(\left|\hat{\alpha}\left(s_{i}, v_{j}\right)\right|\right), \\
\left(\hat{s}_{2}, \hat{v}_{2}\right)=\underset{\left(s_{i}, v_{j}\right) \in\left(\mathcal{I}_{s}, \mathcal{I}_{v}\right)}{\arg \max }\left(\frac{\left|\mathbf{a}^{H}\left(s_{i}, v_{j}\right) \hat{\mathbf{P}}_{1}^{\perp} \mathbf{y}^{p d}\right|}{\left\|\hat{\mathbf{P}}_{1}^{\perp} \mathbf{a}\left(s_{i}, v_{j}\right)\right\|}\right) .
\end{gathered}
$$

$\hat{\mathbf{P}}_{1}^{\perp}$ is the projector onto the orthogonal complement of the subspace spanned by the steering vector of the first scatterer, $\mathbf{a}^{H}\left(\hat{s}_{1}, \hat{v}_{1}\right)$ :

$$
\hat{\mathbf{P}}_{1}^{\perp}=\mathbf{I}_{M}-\frac{\mathbf{a}\left(\hat{s}_{1}, \hat{v}_{1}\right) \mathbf{a}^{H}\left(\hat{s}_{1}, \hat{v}_{1}\right)}{M} .
$$

To distinguish between the aforementioned hypotheses, we use the sequential generalized likelihood ratio test with cancellation (SGLRTC), as proposed by Pauciullo et al. in [29]. SGLRTC compares the normalized energies of the potential scatterers against preselected thresholds. For details on SGLRTC the reader is referred to [29] and to our earlier works in [31], [79], [80].

\section{DATA STACK}

The interferometric data stack used in this work comprises 32 Cosmo-SkyMed stripmap SAR images. These images were acquired during 2008-2013 in repeated passes over Matter Valley in the Swiss Alps, as shown in Fig. 3a. As the region is mostly snow-covered during the winters, we only use acquisitions from the summers. The region is known to have many dormant as well as active landslides, rockslides and rockfalls [81], [82]. The topography varies from around 1200 $\mathrm{m}$ to $4000 \mathrm{~m}$ a.s.l., from the valleys to the mountaintops. There are several slopes with considerable parts free of vegetation or sparsely covered with patches of alpine grass. An average SAR intensity image is shown in Fig. 3b. Due to the drastic height variations, most of the valleys are completely or partly in layover of the nearby mountains.
The region of interest (ROI) selected for tomographic investigation is the area around Zermatt, as marked in red in the full scene shown in Fig. 2b. Compared to the other settlements in the full scene, the Zermatt village is more built-up and therefore, more PS candidates are expected here. A zoom-in of the ROI is shown in Fig. 2c, where the top image is the SAR intensity image and the bottom image is an optical perspective from Google Earth. The height difference between the valley floor and the mountain top is on the order of a kilometer. We can see vegetation stretched over the slope in near range. As to the one in far range, which casts the layover, we can observe some bare rocks on the mountainside and a few ridges which may exhibit long term coherence.

The distribution of the temporal and spatial baselines, and the corresponding point spread function (PSF), are shown in Fig. 4. Due to the non-uniformity of the baselines, the PSF is distorted with several irregularly-spread side-lobes. The total orthogonal baseline, i.e. the tomographic synthetic aperture in elevation, $B^{\perp}=1844 \mathrm{~m}$. The resolution in the elevation, $\delta_{s}$, had the aperture extended over uniform samples of interferometric baselines, would be as follows [11], [83]:

$$
\delta_{s}=\frac{\lambda r_{m}}{2 B^{\perp}}=6.6 \mathrm{~m} \text {. }
$$

where $\lambda=3.12 \mathrm{~cm}$ and $r_{m}=701 \mathrm{~km}$. The resolution projected in height is $3.0 \mathrm{~m}$, corresponding to the incidence angle of $27.3^{\circ}$ for the reference layer. This meter-level resolution is conducive to fine-geocoding of the detected scatterers as well as for the detection of closely-spaced multiple scatterers in layover. At the same time, considering the layover of the mountainside and the valley, the extent of the elevation to be observed $\left(\mathcal{I}_{s}\right)$ for tomographic inversion is on the order of several hundred meters. A resolution too fine may require exceedingly high sampling rates along the elevation to accommodate the possibility of detecting double scatterers between the valley and the mountainside, or even single scatterers along the mountainside when they are dominant over the backscattering from the valley. As a consequence, the computational load is increased.

Another inopportune implication of a large tomographic aperture in mountainous regions is the possibility of range migration of the target from a given range bin to the next or previous. Due to the different viewing angles in each pass, the iso-range curves for each sensor at the given range bin are not identical. Coregistration of the stack is, in principle, tying these iso-range curves at a specific height. In our case, as we use a terrain-based coregistration with the help of an external DEM, this height can be considered to be close to the DEM height. As we apply tomographic focusing at locations significantly farther from this height, the difference between the iso-range curves may exceed the dimension of the range resolution. Consequently, the true SLC signal value may actually migrate to the neighboring range bin. With larger baselines, this limiting point would occur at shorter height changes. Considering the total aperture as the worst-case of baseline diversity, the limit on the observed elevation extent to avoid this range migration is [31], [64]:

$$
\mathcal{I}_{s} \ll \frac{\delta_{r} r_{m}}{B^{\perp}}=663 \mathrm{~m} .
$$




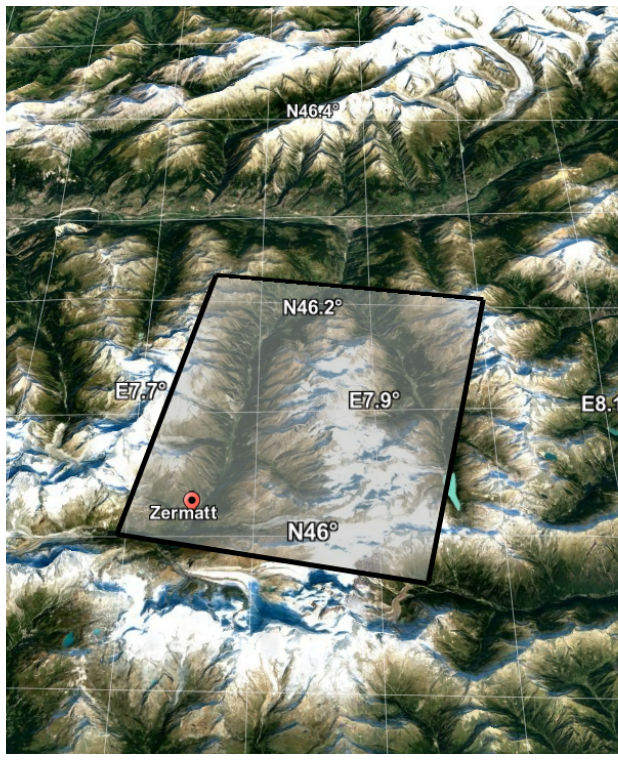

(a)

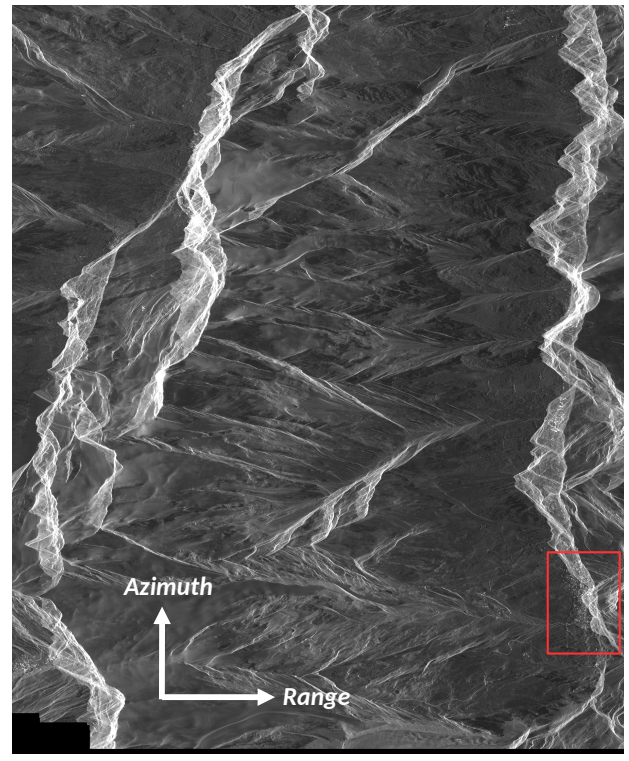

(b)
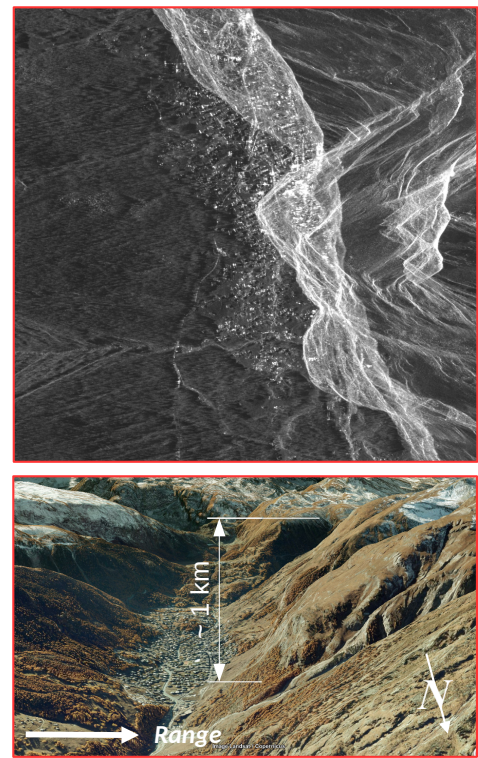

(c)

Figure 3. Cosmo-SkyMed acquisitions over Matter Valley in the Swiss Alps. (a) The footprint of the acquisitions shown on top of the topography in map coordinates. (b) Average SAR intensity image in radar coordinates. The region of interest (ROI) for tomographic analysis is Zermatt village and the surroundings, as indicated with the red-colored rectangle. (c, top) Average SAR intensity image of the ROI. (c, bottom) An optical perspective of the ROI, dated August 30, 2009, (c) Google Earth.
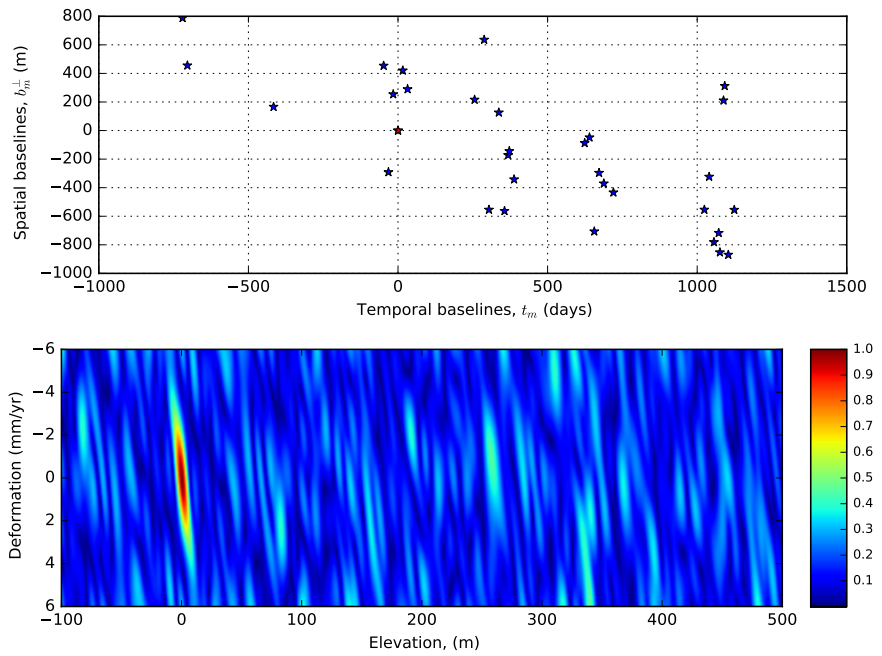

Figure 4. Data characteristics. (Top) Distribution of spatial (orthogonal component) and temporal baselines. (Bottom) Two-dimensional point spread function in elevation-deformation plane, for the baseline distribution shown above.

where $\delta_{r}=1.5 \mathrm{~m}$ is the range resolution. Projecting vertically, the limiting height difference is nearly $300 \mathrm{~m}$. In other words, it may not be possible to detect potential double scatterers whose separation in height is approaching $300 \mathrm{~m}$. To overcome this limitation, a simple strategy is to select a subset comprising smaller baselines; for example, restricting the maximum baselines within $\pm 400 \mathrm{~m}$ with the same reference acquisition provides an improvement by raising the limit to $822 \mathrm{~m}$. The drawback is the reduction in the size of the stack (in this case to 17) which in turn reduces the achievable precision in the estimates obtained with tomography [65],
[84]. Incidentally, tomographic focusing with time domain back-projection (TDBP) [85], [86] or other range-migration correction techniques can be used to obviate the limitation on the observed extent, but it falls outside the focus of this work.

\section{Results}

This section presents the results obtained with the application of the proposed methodology on the interferometric data stack introduced in the previous section.

\section{A. PSI processing \& regression-kriging}

The distribution of the PS identified in the interferometric processing with IPTA is shown in Fig. 5. The left sub-figure shows the distribution over the full scene, while the right subfigure shows the PS found in the Zermatt valley. For each PS, the final solution obtained after several iterations comprises the estimates of the residual topography, deformation in the LOS and atmospheric phases. A linear model for height-dependence of atmospheric phase has been applied using an external DEM at $5 \times 5 \mathrm{~m}$ posting. The quality of the PSI solution is assessed in terms of the standard deviation of the residual phase, $\sigma_{\text {res }}$ for each candidate PS. Only the candidates with $\sigma_{\text {res }}<0.9$ rad are qualified as PS.

In this investigation, we are specifically interested in the atmospheric phases of the PS. After geocoding, the trends in the atmospheric phases along easting, northing and height are estimated and removed for each interferometric layer. The residue of the regression fit, as shown in Fig. 6, is subjected to a variogram analysis. The empirical semivariance is fit with a parametric model, and thereby a spatial covariance function is selected for each layer. The regression coefficients and the covariance function allow universal kriging prediction, cf. eq. 

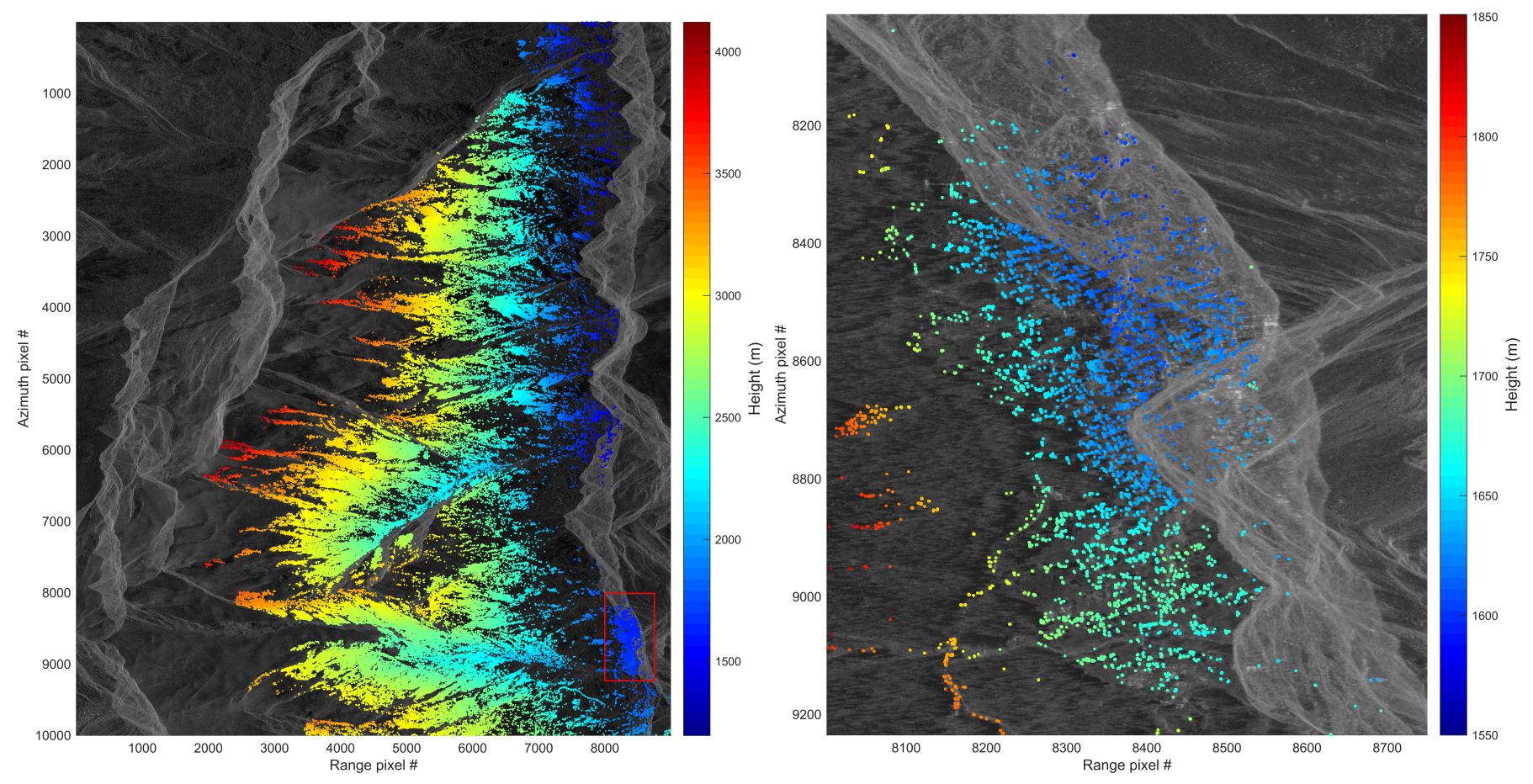

Figure 5. The distribution of the persistent scatterers (PS) identified in the interferometric processing. Left: PS over the entire scene. Right: PS identified in the region of interest for subsequent tomographic processing.

17. In order to assess the precision of the kriging predictions in the ROI, we perform kriging at a set of random locations. These random locations are generated as follows. 500 eastingnorthing pairs are randomly initialized within a lateral radius of $300 \mathrm{~m}$ around the reference point, at the fixed height of $1600 \mathrm{~m}$ (valley floor). To allow vertical sampling, the height interval of $[1600,2900]$ is divided into 15 bins. For each random easting-northing pair, we perform kriging predictions at each height bin. Fig. 7 shows the standard error, $\sigma_{e}$ for these predictions against increasing height, for each interferometric layer in the stack. The black dots represent the mean values of the standard errors, while the blue vertical bars indicate the minimum and the maximum error for the random locations at each height bin. The subset of the PS used for the kriging setup are shown in the rightmost sub-figure in the first row. They are color-coded in height. The circle indicates the region in which the random locations are initialized.

\section{B. Tomography}

Single and double scatterers obtained with BF-based differential tomography and SGLRTC are shown in Fig. 8 in radar coordinates. The height and deformation velocity of the scatterers have been simultaneously estimated. The thresholds of detection are set at 0.48 [29], [31]. The detected scatterers are geocoded and projected in Google Earth, as shown in Fig. 9. The PS found in the Zermatt valley are also projected for comparison. It can be seen that some single scatterers, as encircled in white, are detected around $230 \mathrm{~m}$ above the valley floor along the mountainside only in the tomography solution. A few double scatterers are also detected, but nearly all of them are situated within the built-up area in the valley. Fig.
10 shows the squared 2D reflectivity in the height-deformation plane for a layover-affected pixel in the valley. It potentially contains coherent backscatter from a structure in the valley floor, as well as from rocky edges on the mountainside. The reflectivity is retrieved in three cases: I - no atmospheric correction is applied, II - a single atmospheric correction is applied, estimated by filtering the atmospheric phases of neighboring PS in the valley floor, and III - height-dependent regression kriging-based atmospheric correction is applied as proposed in this work (eq. 17, 22 and 23). Multiple scatterers are detected in the third case, as marked in white.

\section{DISCUSSION}

This section provides an itemized discussion of the results presented in the previous section.

\section{A. PSI processing \& regression-kriging}

The natural terrain in mountainous regions generally limits the prevalence of coherent scatterers. Moreover, the ruggedness of the topography results in frequent layovers which are also typically rejected in the interferometric processing. Consequently, the PS identified in the interferometric processing, as shown in Fig. 5, correspond mostly to bare rocks and manmade structures in layover-free areas. Since the atmospheric phases for these PS are subsequently used in the kriging setup, low quality PS may lead to noise propagation in the prediction of the atmospheric phase at unobserved locations. Therefore, a quality control is imperative. In this work, we used only those PS candidates whose residual phase standard deviation is below $0.9 \mathrm{rad}$. A more strict threshold can further reduce the 
Residue of the regression fit, $\varepsilon(\boldsymbol{x})$ (geocoded)

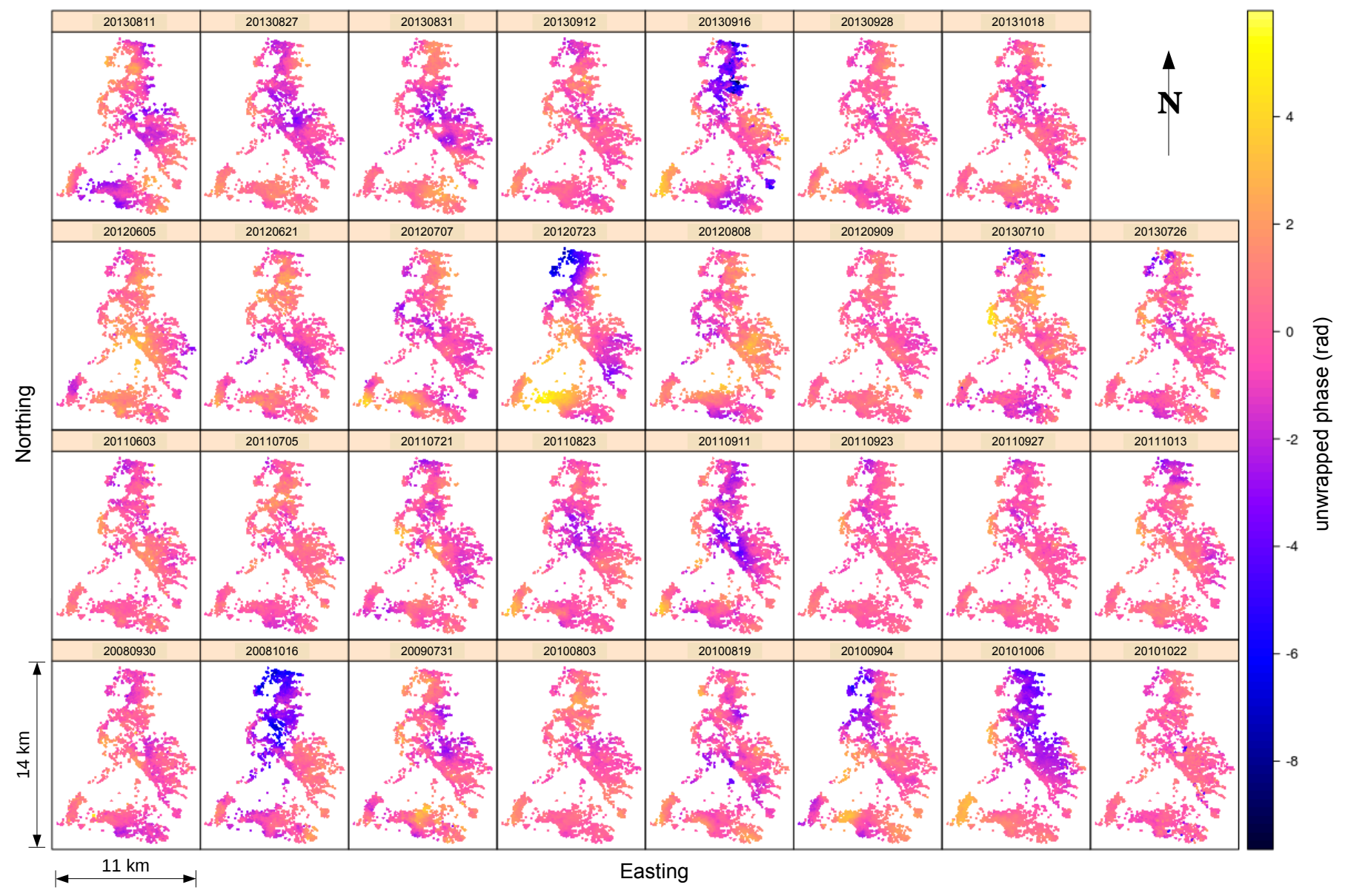

Figure 6. Residue of the multiple linear regression fit of the unwrapped atmospheric phases against easting, northing and height, cf. eq. 13. It is shown here for each interferometric layer in the data stack for the PS identified in the interferometric processing over the full scene, and has been geocoded. The title of the sub-figures indicate the date of the SAR acquisition in YYYYMMDD format. Interferogram network is set up with reference to the acquisition on 20100920 .

possibility of noise propagation, but at the expense of reduced coverage.

The residue of the regression fit at geocoded PS locations is shown in Fig. 6. In some interferometric layers, the phases are more smooth than the others. It is not unexpected as refractivity changes between the reference acquisition and the other acquisitions in the stack depend on meteorological conditions which can be more variable at times. Nonetheless, spatial correlation up to a certain extent can be observed in each case, which encourages the use of kriging interpolation.

It can be seen in Fig. 5 (left) that the Zermatt valley floor is partly covered with the layover cast by the adjoining mountain. A few PS are found even in the layover, representing those pixels where one among the other scattering contributions is dominant. Nearly all of such PS are situated within the valley, and there is no coverage along the mountainside or the top. Since the PS distribution also represents the sampling of the 3-D atmosphere that is subsequently interpolated with kriging, lack of PS at high altitudes is inopportune as it may lead to high predication error variance. To investigate it, we randomly initialized 500 locations within a $300 \mathrm{~m}$ radius of the reference point, and performed kriging predictions at various heights for these locations. The kriging predication errors, as shown in Fig. 7, are increasing with height in each interferometric layer. The top of the mountain within the ROI is around $2800 \mathrm{~m}$. At this height bin, the on-average prediction error is varying between $[0.4,1.4] \mathrm{rad}$ among the different interferometric layers. For the majority of the layers, it is more than $0.85 \mathrm{rad}$ (median value), which can indeed be a limiting factor in detecting coherent scatterers at high altitudes [13]. Moreover, these figures only represent the prediction error due to the kriging setup and the spatial covariance functions. Any errors in the prior estimation of the atmospheric phase for the PS, or their propagation during the PSI processing, are not accounted for.

\section{B. Tomography}

Single and double scatterers, as shown in Fig. 8 and 9, are obtained by setting the detection thresholds such that there are no obvious false alarms over decorrelated areas (e.g. forest) or detections at impossible locations (mid-air, below ground) after geocoding. A higher coverage is observed in the layover region relative to the PSI solution, though the apparent gain 


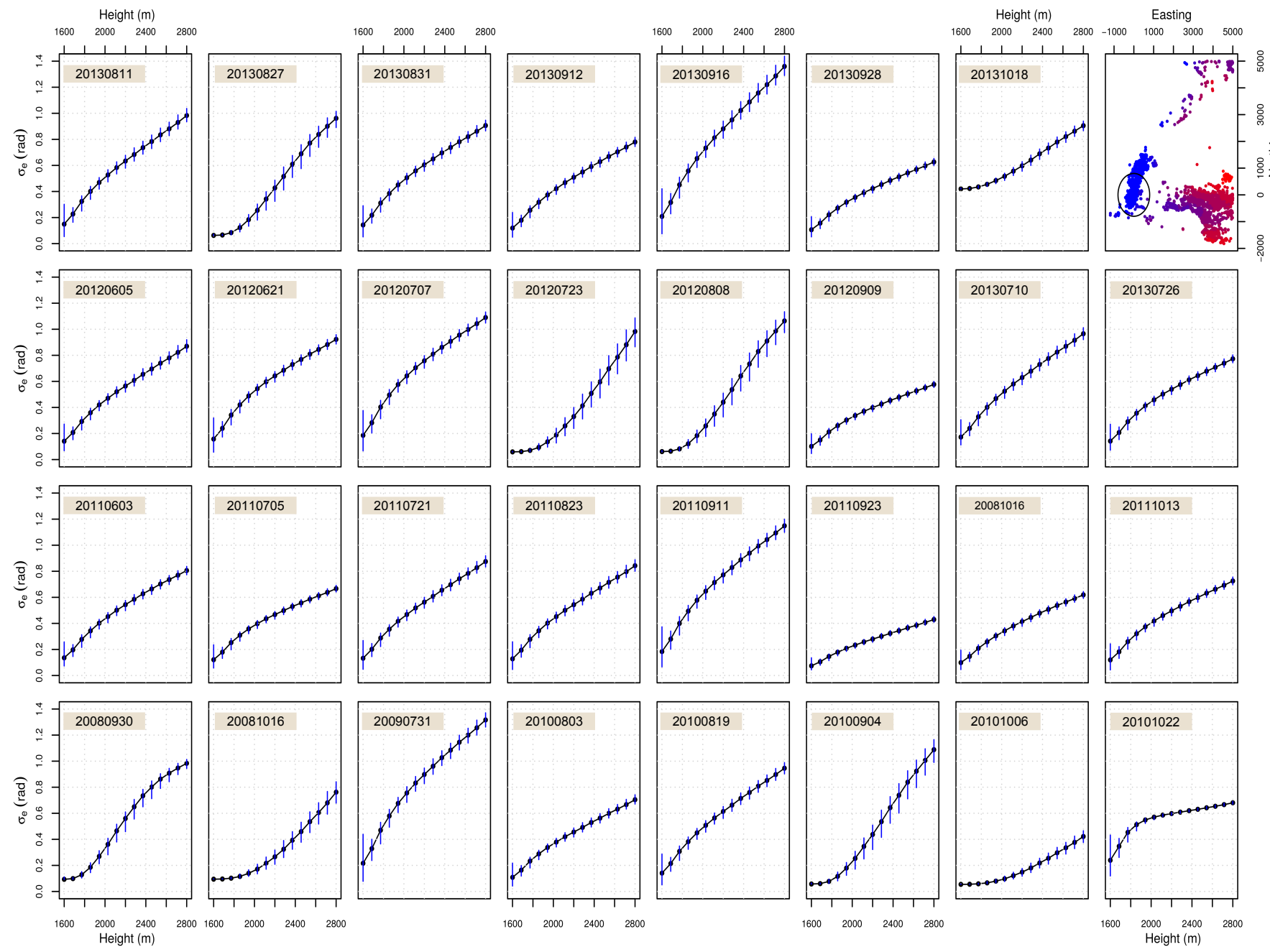

Figure 7. Sub-figures 1-31: Kriging predication standard error, $\sigma_{e}$ at different heights for 500 random locations in Zermatt valley, for each interferometric layer in the stack. The black dots represent the mean values of $\sigma_{e}$ for the random locations at each height bin, while the blue vertical lines indicate the range between the minimum and the maximum. The dates of the SAR acquisitions are mentioned in YYYYMMDD format. Interferograms are referenced to the acquisition on 20100920. Top-rightmost sub-figure: Geocoded PS distribution in the valley, as used for the design matrix in the kriging setup. The random locations are initialized within $300 \mathrm{~m}$ radius (marked in circle) of the reference point. The easting and northing coordinates are given relative to the reference point. The color coding represents height variation, from 1550 (blue) to 2900 (red).

in coverage remains to be assessed vis-à-vis quality of the detected scatterers relative to the quality of the PS.

A group of single scatterers are detected on the mountainside, around $230 \mathrm{~m}$ above the valley floor, where no PS were found with the PSI processing. These scatterers are found on vegetation-free patches of rock, as can be seen in Fig. 9 (right). The reason they have not been identified in the prior PSI processing may be that they are in layover. However, since they are single dominant scatterers, there is possibly another explanation. In our PSI processing, although a linear model for height-dependence of atmospheric phase has been used, only a single correction is applied corresponding to the reference/DEM height of the pixel (as used for the model fit). In case of the aforementioned scatterers, this reference height corresponded to the valley - rather than the mountainside - which would have been appropriate had the dominant scattering originated from the valley. The scatterers would still be detected at the correct location in terms of residual topography correction with PSI processing, if only there was no substantial height-dependence of the atmospheric phase. In this case, the scatterers along the mountainside were $230 \mathrm{~m}$ higher above the reference, and height-dependence of the atmosphere was not negligible. These limitations prevented the detection of these scatterers with the PSI processing. In short, these limitations are overcome with the methodology proposed in this work: the tomographic inversion retrieves the correct residual topography relative to the reference height as the atmospheric phases are corrected simultaneously while focusing along the elevation axis.

Fig. 10 presents the case of another layover-affected pixel, which was not detected as a PS. When no atmospheric correction was applied, no coherent scatterer was detected. On applying a single correction estimated with spatial filtering of the atmospheric phases of the neighboring PS, a single 

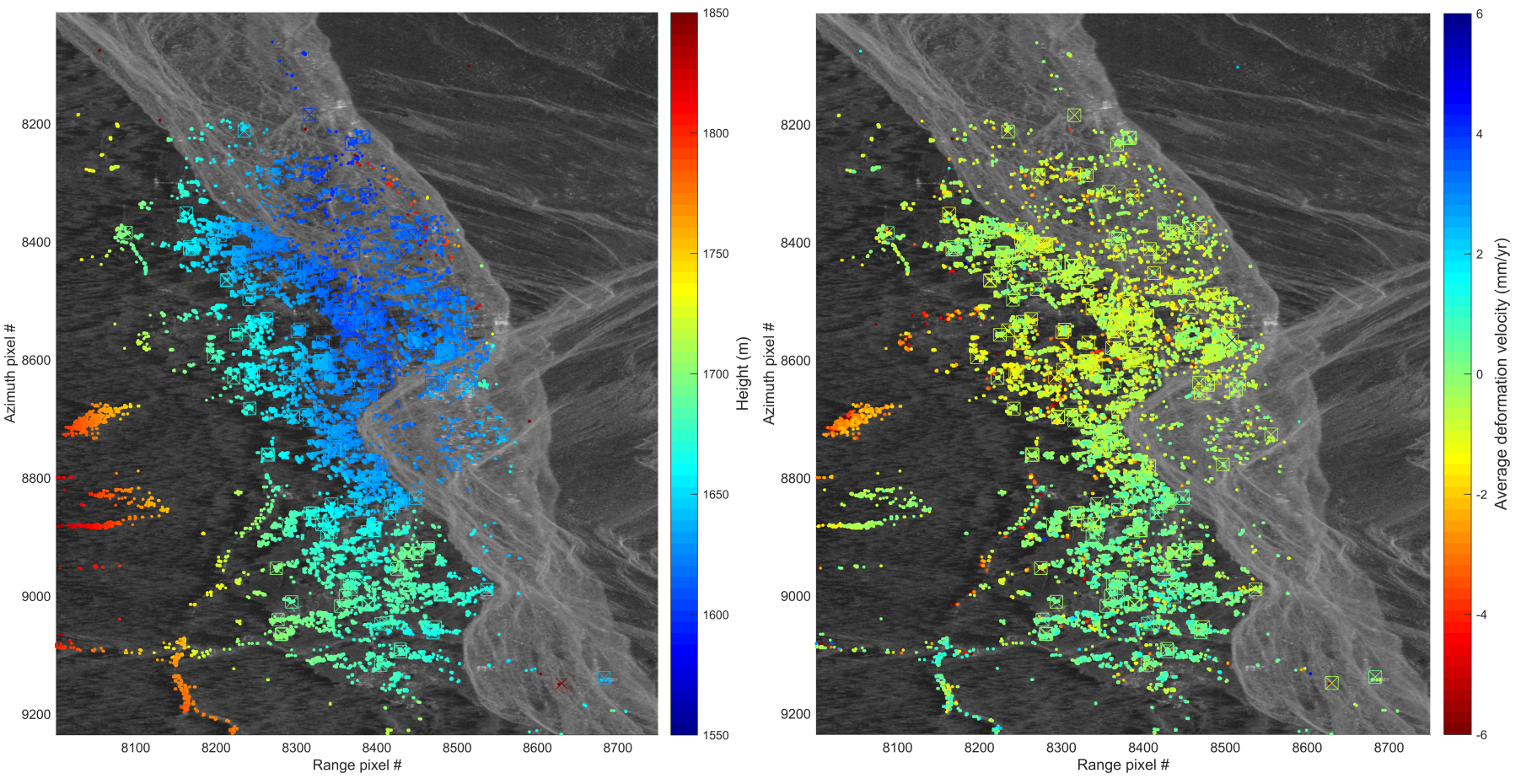

Figure 8. Single and double scatterers obtained with differential SAR tomographic inversion, under the SGLRTC detection strategy with detection thresholds set at 0.48 (referred to [29], [31] for details). Single scatterers are marked with ' $\because$. The first and the second in case of a double scatterer are marked with ' $\square$ ' and ' $X$ ', respectively. The size of the markers for double scatterers is exaggerated for better readability. Left: Estimated height a.s.l. in m. Right: Estimated average deformation velocity in $\mathrm{mm} / \mathrm{yr}$.
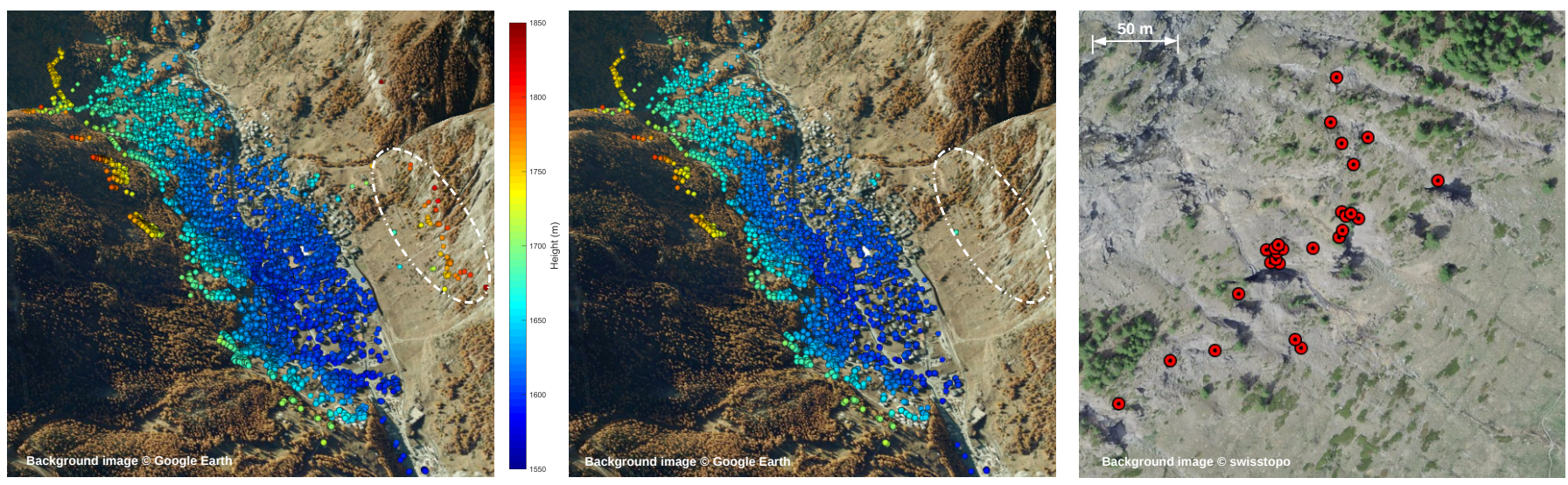

Figure 9. Geocoded scatterers. Left: Single and double scatterers obtained with tomography. Regression kriging-based height-dependent atmospheric corrections have been applied. A very few double scatterers have been detected. Nearly all of them are situated within the built-up area in the valley floor. The color-coding represents the estimated height. Middle: PS identified in the prior PSI processing, shown with the same color-coding as in the left sub-figure. Right: Single scatterers detected with tomography on the mountainside (enclosed in white boundary in the left sub-figure) around $230 \mathrm{~m}$ above the valley floor.

scatterer is detected corresponding to a structure in the valley. When a height-dependent atmospheric correction is applied, we observe multiple scatterers appearing around the mountainside, around $200 \mathrm{~m}$ above the valley - the layover is resolved. These results also substantiate the applicability and usefulness of the proposed methodology.

Notwithstanding, we do not observe any substantial number of single, double or higher order scatterers at higher altitudes along the mountainside or the top. A very few double scatterers are detected, merely 274 , and nearly all of them are situated within the built-up area in the valley floor. Firstly, there may be a lack of coherent point-like scattering due to large pixel- area or unavoidable temporal decorrelation. Another factor may be the increasing atmospheric phase predication error with increasing height. SAR tomography requires high phase stability. Referring to the relevant theoretical formulations in [13], for a data stack comprising 32 acquisitions, in order to achieve a probability of detection better than $95 \%$ while keeping the probability of false alarm under $10^{-4}$, the precision of the overall phase correction (for the atmospheric disturbances as well as any other source of phase instability) needs to be better than $1.0 \mathrm{rad}$ for a single scatterer and $0.85 \mathrm{rad}$ for a double scatterer, on average. Given the fact that just the kriging predication error approaches $0.85 \mathrm{rad}$ 


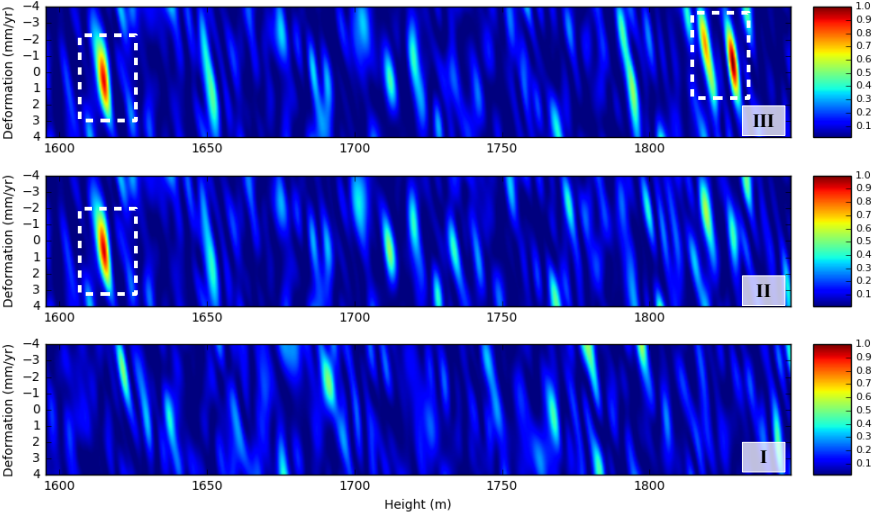

Figure 10. 2D squared reflectivity in the height-deformation plane, retrieved with BF-based tomographic inversion, for a layover-affected pixel. Subfigure I: No atmospheric correction has been applied. Sub-figure II: A single correction is applied using the spatially filtered atmospheric phase from neighboring PS in the valley. Sub-figure III: Regression kriging-based heightdependent atmospheric corrections are applied. Multiple scatterers are detected in this case, as marked in white.

around the mountain top for most of the interferometric layers, the predicted atmospheric corrections are (presumably) not precise enough to detect coherent scatterers at high altitudes [13]. Further investigations are needed to understand how the errors in the prior estimation of atmospheric phases for the PS propagate into errors in the kriging predictions at unobserved (non-PS) locations.

The possibility of target range migration with increasing height can also be a reason for lack of scatterers at high altitudes. In order to alleviate its impact, we experimented with a subset of 17 acquisitions from the data stack such that the orthogonal component of the spatial baselines are within $\pm 400 \mathrm{~m}$. However, no improvement is observed in the results. Arguably, any potential improvement is offset by the increase in phase stability requirements due to the reduction in the size of the stack [13].

\section{CONCLUSION \& OUTLOOK}

This paper has discussed the role of atmospheric phases as one of the key limiting factors in the applicability of SAR tomography in mountainous regions. Given the possibility that the scatterers in layover may be separated in height by several hundreds of meters, the need to perform height-dependent atmospheric corrections is imperative. This paper has also proposed a methodology to correct for the atmospheric phases and incorporate it within tomographic processing. It is a datadriven approach that entails a prior PSI processing. Atmospheric phases are predicted at unobserved (non-PS) locations using universal kriging with 3-D spatial map coordinates as the regressors. The phase corrections are applied at each 3-D point of interest while focusing along the elevation axis. The proposed methodology has been experimented on a data stack comprising 32 Cosmo-SkyMed acquisitions over Matter Valley in the Swiss Alps. Differential SAR tomography is applied in a valley partly covered by the layover of the adjoining mountain. Apart from the single scatterers detected on the man-made structures in the valley, some single scatterers are detected along the mountainside around $230 \mathrm{~m}$ above the valley floor, where no PS were found in the prior PSI processing. The case of another layover-affected pixel which comprises multiple scatterers is presented: it was rejected during the PSI processing, but the proposed tomographic processing resolved the layover and separated the individual scatterers. These results substantiate the usefulness of the kriging-based atmospheric corrections introduced in the paper.

Nonetheless, increase in coverage in terms of layover separations remains limited as only a few double scatterers have been detected, and nearly all of them belong to the layovers occurring in the built-up area in the valley floor. Apart from the fact that large pixel-area squeezed in a few range bins limits point-like scattering, the sparsity of the PS distribution at high altitudes has also been a limiting factor. The precision of the atmospheric phase corrections gets lower for locations farther from the distribution which in turn hinders scatterer detection.

This paper has also hinted at factors other than atmospheric disturbances that may limit the detection of single and double scatterers at high altitudes, such as the possibility of range migration due to large elevation extents that may need to be observed in rugged mountainous regions. Choosing a subset of spatial baselines such that the total orthogonal baseline is not too large to cause significant range migration may in turn increase the requirement on the precision of the phase calibration to achieve a certain probability of detection. Therefore, future investigations should explore strategies for the correction of range-cell migration while focusing along the elevation, especially when layover separation is attempted over large elevation extents.

\section{ACKNOWLEDGMENTS}

This research project has been partially funded by the Swiss Space Office, State Secretariat for Education and Research of the Swiss Confederation (SER/SSO), in the frame of the "Space Technology Studies' MdP2012 project "GAMMA software module for spaceborne SAR tomography". The Digital Elevation Model (DEM) used in this work is (C) swisstopo (JD100042). Variogram analysis and modeling has been performed using the Gstat package [78], [87]. The data stack used in this work is a COSMO-SkyMed Product - OASI - Agenzia Spaziale Italiana - (2013). All Rights Reserved.

\section{REFERENCES}

[1] F. J. Meyer, "Performance requirements for ionospheric correction of low-frequency SAR data," IEEE Trans. Geosci. Remote Sens., vol. 49, no. 10 , pp. 3694-3702, 2011. I

[2] G. Gomba, A. Parizzi, F. D. Zan, M. Eineder, and R. Bamler, "Toward operational compensation of ionospheric effects in SAR interferograms: The split-spectrum method," IEEE Trans. Geosci. Remote Sens., vol. 54, pp. 1446-1461, March 2016. I

[3] R. F. Hanssen, Radar interferometry: Data interpretation and error analysis, vol. 2. Springer Science \& Business Media, 2001. I, I-B, II, II-B

[4] N. Blaunstein and C. Christodoulou, Effects of the Troposphere on Radio Propagation, pp. 175-236. John Wiley \& Sons, Inc., 2006. I, II-B

[5] H. A. Zebker, P. A. Rosen, and S. Hensley, "Atmospheric effects in interferometric synthetic aperture radar surface deformation and topographic maps," J. Geophys. Res. B: Solid Earth, vol. 102, no. B4, pp. 7547-7563, 1997. I, I-B 
[6] C. Colesanti, A. Ferretti, F. Novali, C. Prati, and F. Rocca, "SAR monitoring of progressive and seasonal ground deformation using the permanent scatterers technique," IEEE Trans. Geosci. Remote Sens., vol. 41, pp. 1685-1701, Jul. 2003. I, I-B

[7] P. Agram and M. Simons, "A noise model for InSAR time series," $J$. Geophys. Res. B: Solid Earth, vol. 120, no. 4, pp. 2752-2771, 2015. I

[8] D. Bekaert, R. Walters, T. Wright, A. Hooper, and D. Parker, "Statistical comparison of InSAR tropospheric correction techniques," Remote Sens. Environ., vol. 170, pp. 40-47, 2015. I

[9] A. Reigber and A. Moreira, "First demonstration of airborne SAR tomography using multibaseline L-Band data," IEEE Trans. Geosci. Remote Sens., vol. 38, no. 5, pp. 2142-2152, 2000. I

[10] F. Gini, F. Lombardini, and M. Montanari, "Layover solution in multibaseline SAR interferometry," IEEE Trans. Aerosp. and Electron. Syst., vol. 38, no. 4, pp. 1344-1356, 2002. I

[11] G. Fornaro, F. Serafino, and F. Soldovieri, "Three-dimensional focusing with multipass SAR data," IEEE Trans. Geosci. Remote Sens., vol. 41, pp. 507-517, Mar. 2003. I, IV

[12] O. Frey and E. Meier, "3-D time-domain SAR imaging of a forest using airborne multibaseline data at L-and P-bands," IEEE Trans. Geosci. Remote Sens., vol. 49, no. 10, pp. 3660-3664, 2011. I

[13] S. Tebaldini and A. Guarnieri, "On the role of phase stability in SAR multibaseline applications," IEEE Trans. Geosci. Remote Sens., vol. 48, pp. 2953-2966, July 2010. I, II-B, VI-A, VI-B

[14] O. Frey, I. Hajnsek, and U. Wegmuller, "Spaceborne SAR tomography in urban areas," in Proc. IEEE Int. Geosci. Remote Sens. Symp., pp. 69-72, Jul. 2013. I, I-D

[15] O. Frey, I. Hajnsek, U. Wegmüller, and C. Werner, "SAR tomography based 3-D point cloud extraction of point-like scatterers in urban areas," in Proc. IEEE Int. Geosci. Remote Sens. Symp., pp. 1313-1316, 2014.

[16] G. Fornaro, A. Pauciullo, D. Reale, and S. Verde, "Multilook SAR tomography for 3-D reconstruction and monitoring of single structures applied to COSMO-SKYMED data," IEEE J. Sel. Topics Appl. Earth Observ. Remote Sens., vol. 7, pp. 2776-2785, July 2014. I

[17] S. Tebaldini, F. Rocca, M. M. d'Alessandro, and L. Ferro-Famil, "Phase calibration of airborne tomographic SAR data via phase center double localization," IEEE Trans. Geosci. Remote Sens., vol. 54, no. 3 , pp. 1775-1792, 2016. I

[18] R. F. Hanssen, T. M. Weckwerth, H. A. Zebker, and R. Klees, "Highresolution water vapor mapping from interferometric radar measurements," Science, vol. 283, no. 5406, pp. 1297-1299, 1999. I

[19] R. Goldstein, "Atmospheric limitations to repeat-track radar interferometry," Geophys. Res. Lett., vol. 22, no. 18, pp. 2517-2520, 1995. I, I-B

[20] H. Tarayre and D. Massonnet, "Atmospheric propagation heterogeneities revealed by ERS-1 interferometry," Geophys. Res. Lett., vol. 23, no. 9, pp. 989-992, 1996. I, I-B

[21] R. Hanssen and A. Feijt, "A first quantitative evaluation of atmospheric effects on SAR interferometry," in Proc. FRINGE work. ERS SAR Interf., vol. 406, p. 277, 1997. I

[22] P. Webley, R. Bingley, A. Dodson, G. Wadge, S. Waugh, and I. James, "Atmospheric water vapour correction to InSAR surface motion measurements on mountains: results from a dense GPS network on Mount Etna," Phys. Chem. Earth, vol. 27, no. 4, pp. 363 - 370, 2002. I

[23] A. Ferretti, C. Prati, and F. Rocca, "Permanent scatterers in SAR interferometry," IEEE Trans. Geosci. Remote Sens., vol. 39, no. 1, pp. 8 20, 2001. I-A, I-B

[24] C. Werner, U. Wegmüller, T. Strozzi, and A. Wiesmann, "Interferometric point target analysis for deformation mapping," in Proc. IEEE Int. Geosci. Remote Sens. Symp., pp. 4362-4364, 2003. I-A, I-B, I-E, III-A

[25] P. Blanco-Sánchez, J. J. Mallorquí, S. Duque, and D. Monells, "The Coherent Pixels Technique (CPT): An advanced DInSAR technique for nonlinear deformation monitoring," Pure Appl. Geophys., vol. 165, pp. 1167-1193, Aug. 2008. I-A

[26] A. Hooper, H. Zebker, P. Segall, and B. Kampes, "A new method for measuring deformation on volcanoes and other natural terrains using insar persistent scatterers," Geophys. Res. Lett., vol. 31, no. 23, 2004. I-A

[27] B. M. Kampes and N. Adam, "The STUN algorithm for persistent scatterer interferometry," in FRINGE 2005 Workshop, vol. 610, p. 16, 2006. I-A

[28] F. Lombardini, "Differential tomography: A new framework for SAR interferometry," IEEE Trans. Geosci. Remote Sens., vol. 43, no. 1, pp. 37-44, 2005. I-A, II-A
[29] A. Pauciullo, D. Reale, A. De Maio, and G. Fornaro, "Detection of double scatterers in SAR tomography," IEEE Trans. Geosci. Remote Sens., vol. 50, no. 9, pp. 3567-3586, 2012. I-A, II-A, III-D, III-D, V-B, 8

[30] X. Zhu and R. Bamler, "Compressive sensing for high resolution differential SAR tomography - the SL1MMER algorithm," in Proc. IEEE Int. Geosci. Remote Sens. Symp., pp. 17-20, 2010. I-A, II-A

[31] M. Siddique, U. Wegmuller, I. Hajnsek, and O. Frey, "Single-Look SAR tomography as an add-on to PSI for improved deformation analysis in urban areas," IEEE Trans. Geosci. Remote Sens., vol. 54, pp. 6119-6137, Oct 2016. I-A, I-D, I-D, III-D, III-D, IV, V-B, 8

[32] T. Strozzi, R. Caduff, U. Wegmuller, H. Raetzo, and M. Hauser, "Widespread surface subsidence measured with satellite SAR interferometry in the Swiss alpine range associated with the construction of the Gotthard Base Tunnel," Remote Sens. Environ., vol. 190, pp. 1 - 12, 2017. I-A

[33] C. Barboux, R. Delaloye, T. Strozzi, C. Collet, and H. Raetzo, "TSX InSAR assessment for slope instabilities monitoring in alpine periglacial environment (Western Swiss Alps, Switzerland)," in Proc. ESA FRINGE, pp. 19-23, 2011. I-A

[34] D. Massonnet and K. L. Feigl, "Discrimination of geophysical phenomena in satellite radar interferograms," Geophys. Res. Lett., vol. 22, no. 12, pp. 1537-1540, 1995. I-B

[35] J. Michalakes, J. Dudhia, D. Gill, T. Henderson, J. Klemp, W. Skamarock, and W. Wang, "The weather research and forecast model: software architecture and performance," in Proc. ECMWF Work. on the Use of High Perf. Comp. Meteorology, pp. 156-168, World Scientific: Singapore, 2005. I-B

[36] D. P. Dee et al., "The ERA-Interim reanalysis: Configuration and performance of the data assimilation system," Q. J. R. Meteorolog. Soc., vol. 137 , no. 656 , pp. 553-597, 2011. I-B

[37] G. Nico, R. Tome, J. Catalao, and P. M. A. Miranda, "On the use of the WRF model to mitigate tropospheric phase delay effects in SAR interferograms," IEEE Trans. Geosci. Remote Sens., vol. 49, pp. 49704976, Dec 2011. I-B

[38] X. Cong and M. Eineder, "Volcano deformation measurement using persistent scatterer interferometry with atmospheric delay corrections," in EUSAR 2012; 9th European Conference on Synthetic Aperture Radar, pp. 681-684, April 2012. I-B

[39] S. Liu, R. Hanssen, and A. Mika, "On the value of high-resolution weather models for atmospheric mitigation in sar interferometry," in Proc. IEEE Int. Geosci. Remote Sens. Symp., vol. 2, pp. II-749-II-752, July 2009. I-B

[40] S. Williams, Y. Bock, and P. Fang, "Integrated satellite interferometry: Tropospheric noise, GPS estimates and implications for interferometric synthetic aperture radar products," J. Geophys. Res. Solid Earth, vol. 103, no. B11, pp. 27051-27067, 1998. I-B

[41] Z. Li, E. J. Fielding, P. Cross, and J.-P. Muller, "Interferometric synthetic aperture radar atmospheric correction: GPS topography-dependent turbulence model," J. Geophys. Res. Solid Earth, vol. 111, no. B2, pp. n/a-n/a, 2006. B02404. I-B

[42] F. Onn and H. A. Zebker, "Correction for interferometric synthetic aperture radar atmospheric phase artifacts using time series of zenith wet delay observations from a GPS network," J. Geophys. Res. Solid Earth, vol. 111, no. B9, 2006. I-B

[43] G. Fornaro, N. D'Agostino, R. Giuliani, C. Noviello, D. Reale, and S. Verde, "Assimilation of GPS-derived atmospheric propagation delay in DInSAR data processing," IEEE J. Sel. Topics Appl. Earth Observ. Remote Sens., vol. 8, pp. 784-799, Feb 2015. I-B, II-B, II-B

[44] A. Ferretti, C. Prati, and F. Rocca, "Nonlinear subsidence rate estimation using permanent scatterers in differential SAR interferometry," IEEE Trans. Geosci. Remote Sens., vol. 38, no. 5, pp. 2202-2212, 2000. I-B

[45] U. Wegmuller, D. Walter, V. Spreckels, and C. Werner, "Nonuniform ground motion monitoring with TerraSAR-X persistent scatterer interferometry," IEEE Trans. Geosci. Remote Sens., vol. 48, pp. 895-904, Feb 2010. I-B, III-A

[46] A. Hooper, D. Bekaert, K. Spaans, and M. Arikan, "Recent advances in SAR interferometry time series analysis for measuring crustal deformation," Tectonophysics, vol. 514-517, pp. 1-13, 2012. I-B

[47] A. Hooper, P. Segall, and H. Zebker, "Persistent scatterer interferometric synthetic aperture radar for crustal deformation analysis, with application to Volcán Alcedo, Galápagos,” J. Geophys. Res. Solid Earth, vol. 112, no. B7, 2007. I-B

[48] A. Hooper, "A multi-temporal InSAR method incorporating both persistent scatterer and small baseline approaches," Geophys. Res. Lett., vol. 35 , no. 16,2008 . I-B 
[49] C. W. Wicks, D. Dzurisin, S. Ingebritsen, W. Thatcher, Z. Lu, and J. Iverson, "Magmatic activity beneath the quiescent Three Sisters volcanic center, central Oregon Cascade Range, USA," Geophys. Res. Lett., vol. 29, no. 7, pp. 26-1-26-4, 2002. I-B

[50] J. Elliott, J. Biggs, B. Parsons, and T. Wright, "Insar slip rate determination on the altyn tagh fault, northern tibet, in the presence of topographically correlated atmospheric delays," Geophys. Res. Lett., vol. 35 , no. 12 , 2008. I-B

[51] N. Lin, M. Simons, E. A. Hetland, P. Muse, and C. DiCaprio, "A multiscale approach to estimating topographically correlated propagation delays in radar interferograms," Geochem. Geophys. Geosyst., vol. 11, no. 9, 2010. I-B

[52] R. Iglesias, X. Fabregas, A. Aguasca, J. J. Mallorqui, C. López-Martínez, J. A. Gili, and J. Corominas, "Atmospheric phase screen compensation in ground-based SAR with a multiple-regression model over mountainous regions," IEEE Trans. Geosci. Remote Sens., vol. 52, no. 5, pp. $2436-$ 2449, 2014. I-B, II-B

[53] D. Bekaert, A. Hooper, and T. Wright, "A spatially variable power law tropospheric correction technique for InSAR data," J. Geophys. Res. B: Solid Earth, vol. 120, no. 2, pp. 1345-1356, 2015. I-B, II-B

[54] R. N. Treuhaft and G. E. Lanyi, "The effect of the dynamic wet troposphere on radio interferometric measurements," Radio Science, vol. 22, no. 2, pp. 251-265, 1987. I-B

[55] Z. Li, J.-P. Muller, P. Cross, and E. J. Fielding, "Interferometric synthetic aperture radar (InSAR) atmospheric correction: GPS, Moderate Resolution Imaging Spectroradiometer (MODIS), and InSAR integration," $J$. Geophys. Res. Solid Earth, vol. 110, no. B3, 2005. I-B

[56] S. Jonsson, Modeling volcano and earthquake deformation from satellite radar interferometric observations. Stanford University, 2002. I-B

[57] R. F. Hanssen, "Atmospheric heterogeneities in ERS tandem SAR interferometry," 1998. I-B

[58] T. Emardson, M. Simons, and F. Webb, "Neutral atmospheric delay in interferometric synthetic aperture radar applications: Statistical description and mitigation," J. Geophys. Res. Solid Earth, vol. 108, no. B5, 2003. I-B

[59] R. B. Lohman and M. Simons, "Some thoughts on the use of InSAR data to constrain models of surface deformation: Noise structure and data downsampling," Geochem. Geophys. Geosyst., vol. 6, no. 1, 2005. $\mathrm{I}-\mathrm{B}$

[60] P. O. Jarlemark and T. Emardson, "Strategies for spatial and temporal extrapolation and interpolation of wet delay," J. Geod., vol. 72, no. 6 , pp. 350-355, 1998. I-B

[61] T. R. Emardson and J. M. Johansson, "Spatial interpolation of the atmospheric water vapor content between sites in a ground-based GPS Network," Geophys. Res. Lett., vol. 25, no. 17, pp. 3347-3350, 1998. I-B

[62] V. Janssen, L. Ge, and C. Rizos, "Tropospheric corrections to SAR interferometry from GPS observations," GPS Solutions, vol. 8, no. 3, pp. $140-151,2004$. I-B

[63] G. Fornaro and F. Serafino, "Imaging of single and double scatterers in urban areas via SAR tomography," IEEE Trans. Geosci. Remote Sens. vol. 44 , no. 12 , pp. 3497-3505, 2006. I-D

[64] X. Zhu and R. Bamler, "Very high resolution spaceborne SAR tomography in urban environment," IEEE Trans. Geosci. Remote Sens., vol. 48, no. 12, pp. 4296-4308, 2010. I-D, I-D, II-A, IV

[65] D. Reale, G. Fornaro, and A. Pauciullo, "Extension of 4-D SAR imaging to the monitoring of thermally dilating scatterers," IEEE Trans. Geosci. Remote Sens., vol. 51, no. 12, pp. 5296-5306, 2013. I-D, IV

[66] U. Wegmuller, "Automated terrain corrected SAR geocoding," in Proc. IEEE Int. Geosci. Remote Sens. Symp., vol. 3, pp. 1712-1714, 1999. I-D, III-A

[67] O. Frey, M. Santoro, C. L. Werner, and U. Wegmuller, "DEM-based SAR pixel area estimation for enhanced geocoding refinement and radiometric normalization," IEEE Geosci. Remote Sens. Lett., vol. 10, pp. 48-52, Jan. 2013. I-D, III-A

[68] R. Bivand, E. Pebesma, and V. Gomez-Rubio, Applied spatial data analysis with $R$, vol. 747248717. Springer, 2008. I-E, III-B, III-B, III-B, III-B

[69] F. Lombardini, M. Montanari, and F. Gini, "Reflectivity estimation for multibaseline interferometric radar imaging of layover extended sources," IEEE Trans. Signal Process., vol. 51, pp. 1508-1519, Jun. 2003. II-A

[70] F. Lombardini, F. Cai, and D. Pasculli, "Spaceborne 3-D SAR tomography for analyzing garbled urban scenarios: Single-look superresolution advances and experiments," IEEE J. Sel. Topics Appl. Earth Observ. Remote Sens., vol. 6, no. 2, pp. 960-968, 2013. II-A
[71] G. Fornaro, F. Lombardini, A. Pauciullo, D. Reale, and F. Viviani, "Tomographic processing of interferometric SAR data: Developments, applications, and future research perspectives," IEEE Signal Process. Mag., vol. 31, pp. 41-50, Jul. 2014. II-A, II-A

[72] G. Fornaro and F. Reale, D.and Serafino, "Four-dimensional SAR imaging for height estimation and monitoring of single and double scatterers," IEEE Trans. Geosci. Remote Sens., vol. 47, pp. 224-237, Jan. 2009. II-A

[73] X. Zhu and R. Bamler, "Let's do the time warp: Multicomponent nonlinear motion estimation in differential SAR tomography," IEEE Geosci. Remote Sens. Lett., vol. 8, no. 4, pp. 735-739, 2011. II-A

[74] P. Teatini, L. Tosi, T. Strozzi, L. Carbognin, U. Wegmüller, and F. Rizzetto, "Mapping regional land displacements in the Venice coastland by an integrated monitoring system," Remote Sens. Environ., vol. 98 , no. 4, pp. 403-413, 2005. III-A

[75] C. Werner, U. Wegmuller, A. Wiesmann, and T. Strozzi, "Interferometric point target analysis with JERS-1 L-band SAR data," in Proc. IEEE Int. Geosci. Remote Sens. Symp., vol. 7, pp. 4359-4361 vol.7, Jul. 2003. III-A

[76] U. Wegmuller, O. Frey, and C. Werner, "Point density reduction in persistent scatterer interferometry," in Proc. Europ. Conf. on SAR, pp. 673-676, Apr. 2012. III-A

[77] M. A. Oliver and R. Webster, Basic steps in geostatistics: The variogram and kriging. Springer, 2015. III-B, III-B, III-B

[78] E. J. Pebesma, "Multivariable geostatistics in S: the gstat package," Comput. Geosci., vol. 30, pp. 683-691, 2004. III-B, VII

[79] M. Siddique, I. Hajnsek, U. Wegmüller, and O. Frey, "Towards the integration of SAR tomography and PSI for improved deformation assessment in urban areas," in Proc. ESA FRINGE Workshop, 2015. III-D

[80] M. Siddique, U. Wegmuller, I. Hajnsek, and O. Frey, "SAR tomography as an add-on to PSI: Gain in deformation sampling vis-a-vis quality of the detected scatterers," in Proc. IEEE Int. Geosci. Remote Sens. Symp., pp. $1452-1455$, July 2016 . III-D

[81] T. Strozzi, H. Raetzo, U. Wegmüller, J. Papke, R. Caduff, C. Werner, and A. Wiesmann, "Satellite and terrestrial radar interferometry for the measurement of slope deformation," in Engineering Geology for Society and Territory-Volume 5, pp. 161-165, Springer, 2015. IV

[82] R. Delaloye, S. Morard, C. Barboux, D. Abbet, V. Gruber, M. Riedo, and S. Gachet, "Rapidly moving rock glaciers in Mattertal," Mattertal-ein Tal in Bewegung, p. 30, 2013. IV

[83] A. Reigber and R. Scheiber, "Airborne differential SAR interferometry: First results at L-band," IEEE Trans. Geosci. Remote Sens., vol. 41 pp. 1516-1520, June 2003. IV

[84] R. Bamler, M. Eineder, N. Adam, X. Zhu, and S. Gernhardt, "Interferometric potential of high resolution spaceborne SAR," PhotogrammetrieFernerkundung-Geoinformation, vol. 2009, no. 5, pp. 407-419, 2009. IV

[85] O. Frey, C. Magnard, M. Ruegg, and E. Meier, "Focusing of airborne synthetic aperture radar data from highly nonlinear flight tracks," IEEE Trans. Geosci. Remote Sens., vol. 47, no. 6, pp. 1844-1858, 2009. IV

[86] O. Frey and E. Meier, "Analyzing tomographic SAR data of a forest with respect to frequency, polarization, and focusing technique," IEEE Trans. Geosci. Remote Sens., vol. 49, no. 10, pp. 3648-3659, 2011. IV

[87] E. J. Pebesma and C. G. Wesseling, "Gstat: a program for geostatistical modelling, prediction and simulation," Comput. Geosci., vol. 24, no. 1, pp. $17-31,1998$. VII 


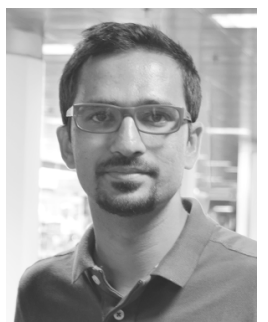

Muhammad Adnan Siddique received the B.E. degree in electronics engineering from the National University of Sciences and Technology, Islamabad, Pakistan, in 2006 and the European Master of Research in Information and Communications Technology (MERIT) joint degree from the Karlsruhe Institute of Technology, Karlsruhe, Germany and Universitat Politcnica de Catalunya (UPC), Barcelona, Spain.

He is currently working toward the Doctoral degree at the Chair of Earth Observation and Remote Sensing, Swiss Federal Institute of Technology (ETH), Zurich, Switzerland. From 2007 to 2008, he worked as a radio-frequency (RF) planning engineer. In Fall 2010, he joined the Department of Electrical Engineering, COMSATS Institute of Information Technology, Lahore, Pakistan, as a Lecturer. His current research interests include synthetic aperture radar tomographic and interferometric techniques for deformation assessment in urban and alpine areas.

Mr. Siddique received the President's Gold Medal for securing the highest cumulative GPA in his class during his undergraduate years. He secured distinction in each semester. He was awarded Erasmus Mundus Scholarship by the European Commission for graduate studies.

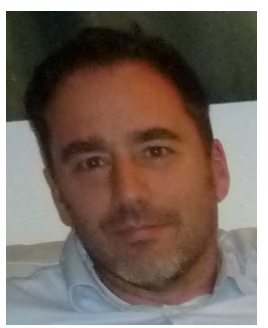

Tazio Strozzi received the M.S. (1993) and Ph.D. (1996) degrees in physics from the University of Berne, Switzerland, for his experimental and theoretical studies on the backscattering characteristics of snow and vegetation. He has been with Gamma Remote Sensing since 1996, where he is responsible for the development of radar remote sensing applications and is manager of a number of research and commercial projects. From 1996 to 1998 he was part-time physics teacher at the Highschool of Bellinzona, Switzerland. From 1999 to 2001 he worked as part-time visiting scientist at the University of Wales, Swansea, UK His current activities include SAR interferometry from space and ground for glacier motion estimation, landslide and rock glacier surveying and subsidence monitoring.

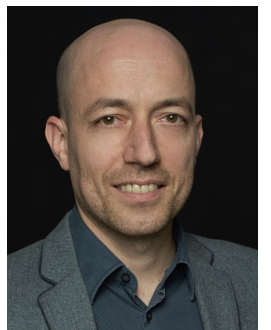

Othmar Frey received the M.Sc. (Hons.) degree in geomatic engineering from the Swiss Federal Institute of Technology (ETH), Zurich, Switzerland, in 2002, and the Dr. sc. nat. (Hons.) degree in radar remote sensing from the University of Zurich, Zurich, Switzerland, in 2010

Othmar Frey is a tenured Senior Researcher and Lecturer at ETH Zurich, Zurich, Switzerland, and also a Senior Scientist at GAMMA Remote Sensing AG, Gümligen, Switzerland. He has been working in this joint academic/industry setting since 2011 . Previously, from 2002 to 2010, he was a Research Associate with the Remote Sensing Laboratories, University of Zurich. His research encompasses 2-D and 3-D (tomographic) synthetic aperture radar (SAR) focusing techniques and interferometric techniques for applications such as 3-D forest mapping, ground deformation monitoring (persistent scatterer interferometry / differential tomography), and profiling the structure of snowpacks, using spaceborne, airborne, and terrestrial radar sensors. He has been active in various national and international research projects, as a PI and project manager as well as a co-investigator. At GAMMA he has also been active in the development of the Gamma Software and in technical consulting related to SAR imaging and interferometric applications. He has been a member of the SAOCOM-CS Science Expert Group with the European Space Agency. Since 2015, he has been the chair of the Swiss Chapter of the IEEE Geoscience and Remote Sensing Society.

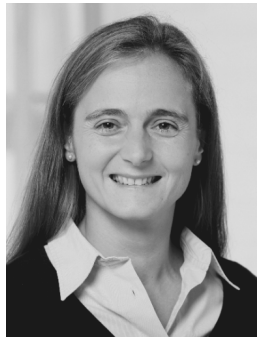

Irena Hajnsek received the Dipl. (Honors) degree from the Free University of Berlin, Berlin, Germany, in 1996 and the Dr. (Hons.) degree from the Friedrich Schiller University of Jena, Jena, Germany, in 2001.

She is currently a Professor of Earth observation and remote sensing with the Institute of Environmental Engineering, Swiss Federal Institute of Technology (ETH) Zurich, Zurich, Switzerland, and the Head of the Polarimetric SAR Interferometry Research Group, Microwaves and Radar Institute, German Aerospace Center, Wessling, Germany. Since 2010, she has been the Science Coordinator of the German satellite mission TanDEM-X. Her main research interests are in electromagnetic propagation and scattering theory, radar polarimetry, SAR and interferometric SAR data processing techniques, and environmental parameter modeling and estimation.

Dr. Hajnsek has been a member of the IEEE Geoscience and Remote Sensing Society Administrative Committee since 2013. She served as the Technical Program Co-chair of the IEEE International Symposium on Geoscience and Remote Remote Sensing in 2012. 